\title{
COMPLEXITY AND PREFERENCE IN ANIMALS AND MEN*
}

\author{
Edward L. Walker, Ph.D. $\dagger$ \\ University of Michigan, Ann Arbor, Mich.
}

Complexity and preference are terms that are relevant to a theory of behavior I presented at the Nebraska Symposium on Motivation several years ago. ${ }^{7}$ The research effort I wish to discuss is based on that theoretical statement. The theory differs very little from an earlier theory published by Dember and Earl (see Dember ${ }^{2}$ ) and it incorporates concepts developed by Berlyne. ${ }^{1}$

The theory contains a small number of terms or concepts and hypotheses which I should like to review briefly in order to make it possible to use them discriminatively in discussing the research. The terms are: Psychological Event, Stimulus Complexity, Psychological Complexity and Optimal Complexity. From these is developed a theory of Preference or choice among psychological events. A hypothesis concerning expected changes in optimal complexity with experience produces an expected set of Changes in Complexity and Preference with Experience. This much is relevant to the experiments to be discussed here. An additional concept, arousal, is in the theory but not germane to these experiments and will not be discussed further.

Psychological Event. This is a term I have used to identify a central unit of activity. A psychological event may be initiated by an external stimulus, but many psychological events have the appearance of spontaneity. A response may result from a psychological event, but many psychological events occur without a visible product. A psychological event may have a conscious representation and, thus, the character of perception, but there are circumstances under which psychological events occur without conscious awareness. Thus the need for such a concept arises from the limitations imposed by the concepts of stimulus, response, perception, and cognition, any or all of which may reflect a psychological event, but none of which is identical.

Stimulus Complexity. This term refers to the complexity of the distal stimulus. The essential characteristic of stimulus complexity-as distinguished from psychological complexity-is that it should be possible to assign a number to a stimulus that represents its complexity and that this value should remain fixed. Often this appears to be no problem, but I believe appearances to be deceiving. With certain kinds of stimulus materials, one can perform a counting operation. One can have stimuli that vary in the number of dots, or polygons that vary in the number of sides. On a priori grounds, it seems reasonable that a card with 50 dots is a more complex stimulus than a card with five, or that a polygon with 20 sides is more complex than a polygon with four sides. Yet, if we subject a set of such materials to psychological scaling procedures, it is very unlikely that there will be perfect agreement between our numerical count and the results of the scaling. One is then tempted to undertake to resolve the discrepancies in terms of factors that were not included in the count-configurational or pattern factors. Alternatively, one can choose one of the two values, the numerical count or the results of psychological scaling, and choose to ignore the other. The question then becomes, which does one choose?

I think there is no question that the choice must be the psychologically derived

- The work reported in this paper was supported by grant no. HD 00904 from the United States Public Health Service.

+ The author is supported by a research career award, K6-MH-21, 868. 
scale. There are two basic reasons. Many important stimuli are not subject to a counting procedure. If I choose to work with abstract paintings as stimuli, there is no simple counting procedure available that yields complexity values having face validity. Even when one can count some aspect of a stimulus set and even when there is a discrepancy between the ordering on the basis of the count and the ordering on the basis of a psychological scaling technique, it is the latter ordering that is likely to be functional in psychological studies.

In a very fundamental way, all quantification, including counting, is a psychological scaling procedure. One's choice, then, should be a psychological scaling procedure that is applicable to all stimuli. It then becomes clear that to achieve a set of complexity values that can be assigned to stimuli and that do not changethus creating the illusion that they belong to the stimuli and not to the observerit is necessary to assign permanent complexity values in terms of mean values for a standard population under standard conditions. This, then, is the definition of stimulus complexity: mean ratings of a reference population under reference conditions.

Psychological Complexity. Psychological complexity is a characteristic of a psychological event. When a psychological event is initiated by a stimulus, psychological complexity is the result of an interaction between the single individual and the distal stimulus. Thus one would expect that same stimulus to result in different psychological complexity values in two different individuals or in the same individual at different times.

Optimal Complexity. In theory, optimal complexity is that degree of psychological complexity that the organism will seek to maintain. For any array of stimuli, the organism will order the stimuli from least to most complex, and the organism can be considered to occupy a position on that scale, his optimal complexity level. The organism will prefer stimuli near his optimum rather than stimuli farther away. -

Preference. The concept of preference is derived directly from the concept of optimal complexity. It is hypothesized that if an organism is asked to choose which of two stimuli he prefers, he will choose that stimulus that is closer to his optimal complexity level. In human subjects, one can ask for a verbal judgment. In animals, one can determine with which of two or more stimuli an animal chooses to interact.

Changes in Complexity and Preference with Experience. The original Dember and Earl theory ${ }^{2}$, as well as my own, postulates that the position the organism occupies on a complexity scale will change with continued experience with the items on the scale, and both predict that the change will be upward toward more complex stimuli. Thus, preferences for musical compositions tend to progress from a liking for simple melodies to a liking for more complex ones, then possibly to classical music, and within classical through a nearly predictable sequence of composers to arrive finally at modern atonal and computer-generated compositions. The progression over a long time span is clearly from the simple to the complex.

Two effects of exposure require some additional specification. There is a shortterm satiation effect of exposure. During a continuous period of exposure, the complexity of a stimulus will undergo reduction. This satiation effect is in part temporary and subject to recovery in the period after exposure. However, recovery is not complete, and the long-term progression to a preference for more and more complex stimuli is the result of the accumulation of these small permanent changes. 
A second effect of exposure is the generalization of change. In the original Dember and Earl theory it was assumed that (in my terms) the psychological complexities of the items on the scale maintained their relative positions during change produced by an exposure to a single stimulus. Thus, it is implied that generalization was complete. In my own statement of 1964 , I operated under the assumption that the items on the scale were independent in the sense that a change in one stimulus produced through interaction with it did not affect the complexity of other stimuli on the scale. Both positions lead to logical difficulties and to a failure to handle much of the sequential choice data. It is probably necessary, therefore, to assume that the effects of exposure to one stimulus are generalized to other stimuli in proportion to distances on the complexity scale.

Two sets of experiments will be discussed. The first set was carried out with human subjects and the second with animals. They will be discussed separately because the special characteristics of the two species (humans and rats) permitted the study of different but related problems.

\section{Stimulus Complexity and Preference in Human Subjects $\ddagger$}

Most of the work that has been done by others in efforts to investigate stimulus preference and complexity has involved either one of two approaches to the selection of stimulus materials. One approach is to select stimulus materials that differ in complexity on the basis of a simple kind of face validity. This approach is handicapped because one cannot plot preference against such nonmetricized stimuli. The second common approach is to generate a set of stimuli with a known physical or quantitative dimensionality.

An alternative approach is to select sets of psychologically interesting stimuli and to subject them to one or more of the psychological scaling techniques. The result might be a set of materials of much more interest than any previously available and a set of materials for which there might be meaningful quantitative dimensions.

In the studies to be discussed in this section, there are generally three aims:

1. To develop sets of visual stimuli with established complexity values based on psychological scaling techniques.

2. To develop methodology for employing these stimuli with human subjects.

3. To investigate the relation of complexity and preference.

The stimuli employed in these studies were derived from four sources:

1. Tartan Patterns. A number of tartan patterns were photographed in black and white to serve as stimuli. These had several virtues as stimuli, but one of the most important was that the number of squares in the pattern gave a physical measure of complexity. It was thus possible to check psychological scaling techniques with a physical scale with this one set of stimuli.

2. Stage Set Designs. A number of drawings of stage set designs were photographed for use as stimuli. They had the virtue of representing well-established styles in set design that differ in apparent complexity.

3. Black and White Graphics. A large set of black and white graphics, all of which were highly representational and symbolic and done by well known artists, was also selected. The graphics had the advantage of being well known to individuals who had studied art, but which were not well known to most individuals.

4. Modern Art. A large set of modern paintings was selected. They were chosen

$\ddagger$ A number of people worked on the stimulus complexity and preference studies from time to time. Much of the work was done by F. Joseph Mortenson, Howard Gadlin, Kathy Nagy, Daneen Hart, and Rivka Fine. 
so that they contained no symbolic material and were generally not identifiable in origin. They are colored prints.

All sets of stimulus materials were either chosen in their original form or photographed and reduced to four-by-six-inch pictures. Each was then given a randomly selected code number that was placed on the back. Finally, each was encased with plastic contact acetate to preserve the original quality during handling.

\section{Tartan Patterns}

The tartan patterns used in these studies were taken from a volume by Innes. 4 From a larger list, the patterns listed in TABLE 1 were selected and tested. As can be seen in the Table, the number of squares in the sample photographed varied from 91 to 2,680 .

In the first study, subjects were asked to rate each of the 21 tartan patterns from simple to complex. They were to use a scale of 1 to 100 , with 1 representing the least complex and 100, the most complex. They were then asked to rate each with respect to how much they liked the pattern. Again they were to use a scale ranging from 1 to 100, with 1 representing the least liked, and 100 the most liked, pattern.

The ratings of complexity are compared to the physical properties of the tartan patterns in FIGURE 1. The number of squares is plotted on a log scale as an approximation of the psychophysical function that is probably involved in judging differences in number of squares. The physical scale and the psychological scale are in fairly good agreement in the Figure. Since there are other possibly physical dimensions in the stimuli, such as grey-scale and relative proportions, this level of agreement seems satisfactory. In fact, it seems possible that the psychological scale might come closer to representing complexity than does the physical scale.

TABLE 1

Tartan Patterns hy Name and Physical Characteristics

\begin{tabular}{clrc}
\hline $\begin{array}{c}\text { Code } \\
\text { Number }\end{array}$ & Tartan & $\begin{array}{c}\text { Number of } \\
\text { Rectangles }\end{array}$ & $\begin{array}{c}\text { Number of } \\
\text { Rectangles } \\
\text { in a Set }\end{array}$ \\
\hline 1 & Royal Stewart & 1,375 & 625 \\
2 & Forbes & 759 & 841 \\
3 & MacArthur & 187 & 81 \\
4 & Skene & 1,452 & 169 \\
5 & Brodie & 102 & 121 \\
6 & Anderson & 2,574 & 1,326 \\
7 & MacDonell of Glengarry & 2,109 & 625 \\
8 & MacKay & 247 & 121 \\
9 & MacAulay & 165 & 110 \\
10 & Kerr & 1,760 & 361 \\
11 & Carnegie & 999 & 625 \\
12 & Ramsey & 375 & 121 \\
13 & Keith & 247 & 81 \\
14 & Sutherland & 984 & 529 \\
15 & MacDonald of Sleat & 273 & 49 \\
16 & Wallace & 165 & 49 \\
17 & MacDonald of Clanranald & 1,718 & 625 \\
18 & Fraser & 247 & 121 \\
19 & Urquart & 1,891 & 729 \\
20 & Montgomerie & 91 & 49 \\
21 & Maclachlan & 405 & 121 \\
\hline \hline
\end{tabular}


Walker: Complexity and Preference

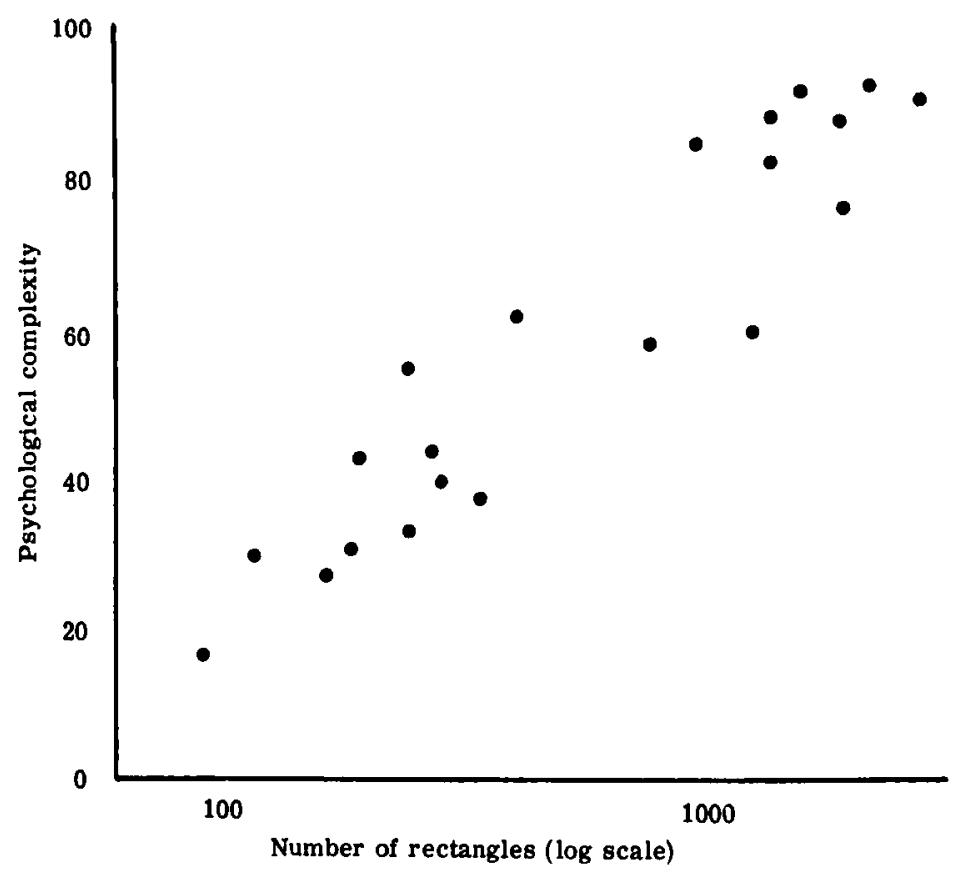

Figure 1. Physical versus psychological scales of complexity in Tartan patterns.

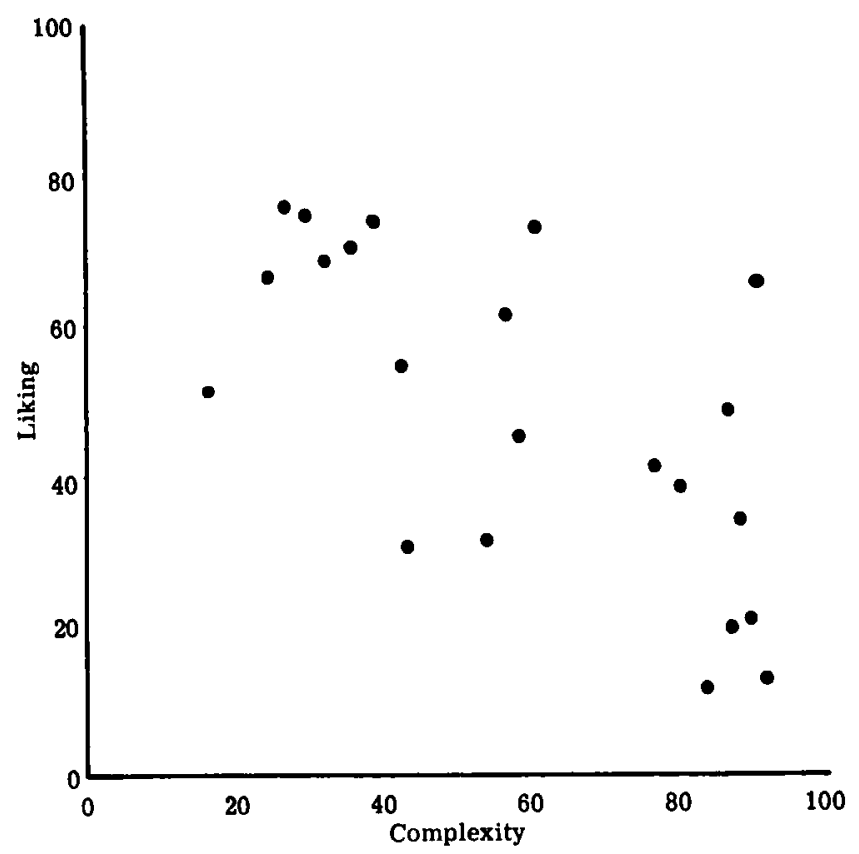

Figure 2. Complexity and liking for Tartan patterns. 
The relation of the psychological scale of complexity and a similar psychological scale of liking is shown in FIGURE 2 . There is an obvious relation between the two measures, with the subjects expressing a greater liking for the simple tartan patterns.

As part of the same study, the subjects were asked to make a second evaluation of the extent to which they liked the tartan patterns. In this case, the patterns were offered in pairs. Subjects were asked to assign a value to the difference in their liking for the two. If the difference in liking was maximum, a value of 10 was to be assigned. If it was minimum, a value of one was to be assigned. Such data can be computer-analyzed, using the Shepard-Kruskal technique.

There are two major advantages to the Shepard-Kruskal scaling and scaling analysis technique. Data collected in this way can be analyzed for any number of dimensions. If a variable such as complexity, liking, or preference is actually multidimensional, the Shepard-Kruskal technique could potentially reveal the fact. The second advantage is that the data collected from a single subject can be subjected to multidimensional analysis. A disadvantage is that a one-dimensional solution is not necessarily the "best" solution. The computer program simply projects a line through the multidimensional space from an arbitrary starting point. It is the best line from that point but not necessarily the best starting point. It is often therefore necessary to ask the computer for an n-dimensional solution and try to match each solution to some criterion. A second disadvantage of the analysis technique is that there are no internal criteria for determining how many dimensions are meaningful. For these reasons, we asked the computer for ten different analyses, specifying numbers of dimensions from 1 to 10 in each case (thus, 55 for each subject). The data from each individual and the group were analyzed separately.

After the subjects had finished their ratings of how well they liked the patterns, they were asked for extensive explanations of the reasons for their liking of a given pattern. Many hours were spent in an effort to match individual Shepard-

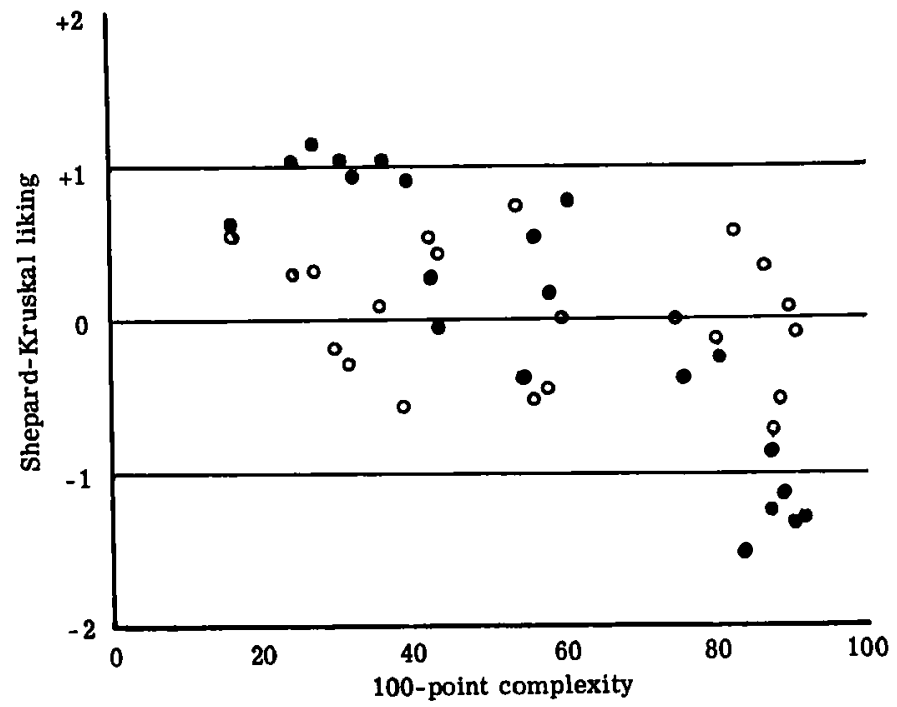

Figure 3. Shepard-Kruskal liking dimensions and complexity. 
Walker: Complexity and Preference
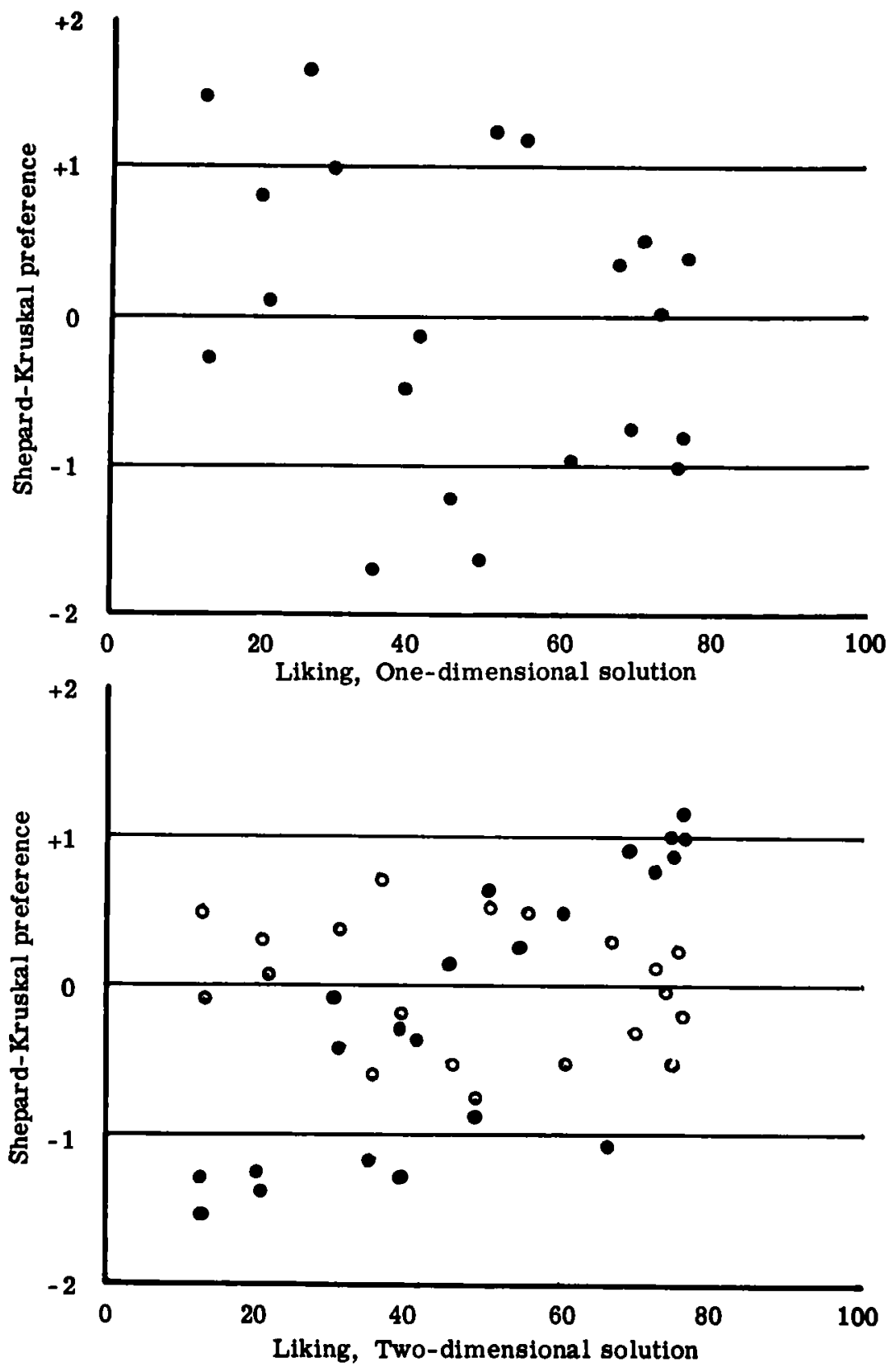

Figure 4. Comparison of two scaling procedures. 
Kruskal dimensions with the verbal protocols, but the effort was fruitless. Liking seemed to be a single dimension, and elaboration of the basis for liking did not improve understanding of the dimension.

Figure 3 is a sample of the Shepard-Kruskal analysis. In this Figure, a twodimensional solution for liking is plotted against the complexity ratings. The first dimension shows little or no relationship between the two variables. The second dimension shows a clear relationship very similar to that shown in FIGURE 2 .

FIGURE 4 is an effort to determine the relationship between the liking scale derived from the simple rating on a 100-point scale and the Shepard-Kruskal analysis of preference. The one-dimensional Shepard-Kruskal solution yields no relationship. When the two dimensions of a two-dimensional solution are plotted, the first dimension shows little or no relationship, while the second dimension shows a rather high correlation.

One further study was carried out with the tartan patterns that was methodological in character. We wished to know what effect the range of stimuli had on ratings of complexity. To determine this, arrangements were made to have subjects rate ten tartan patterns representing the simpler designs, rate ten representing the more complex designs, and rate ten representing the whole range.

The subjects were 15 males and 15 females of college age. They were divided into three groups of ten S's each.

Three sets of stimuli were made up from the tartan patterns that had been rated for complexity in earlier studies. The high complexity set included those rated $1,2,3,4,6,7,8,9,10$, and 12 . The low complexity set included those rated $11,13,14,15,16,17,18,19,20$, and 21 . The full range set included those rated $1,3,6,8,11,12,15,17,19$ and 21 .

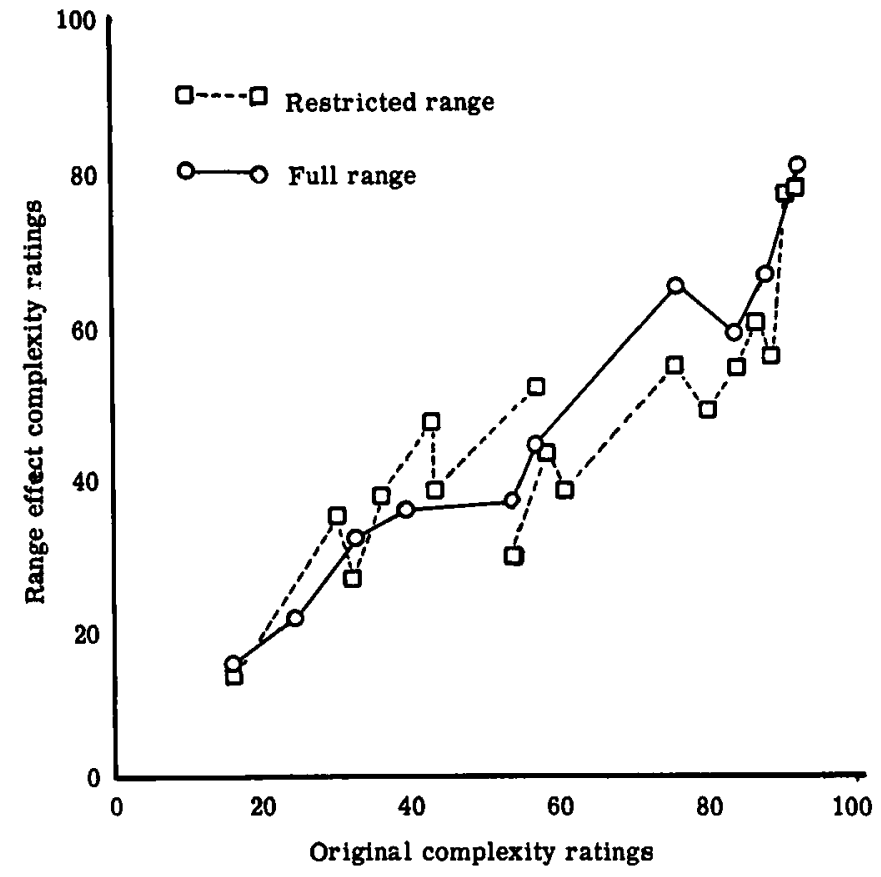

FIGURE 5. Complexity ratings as a function of range of stimuli. 


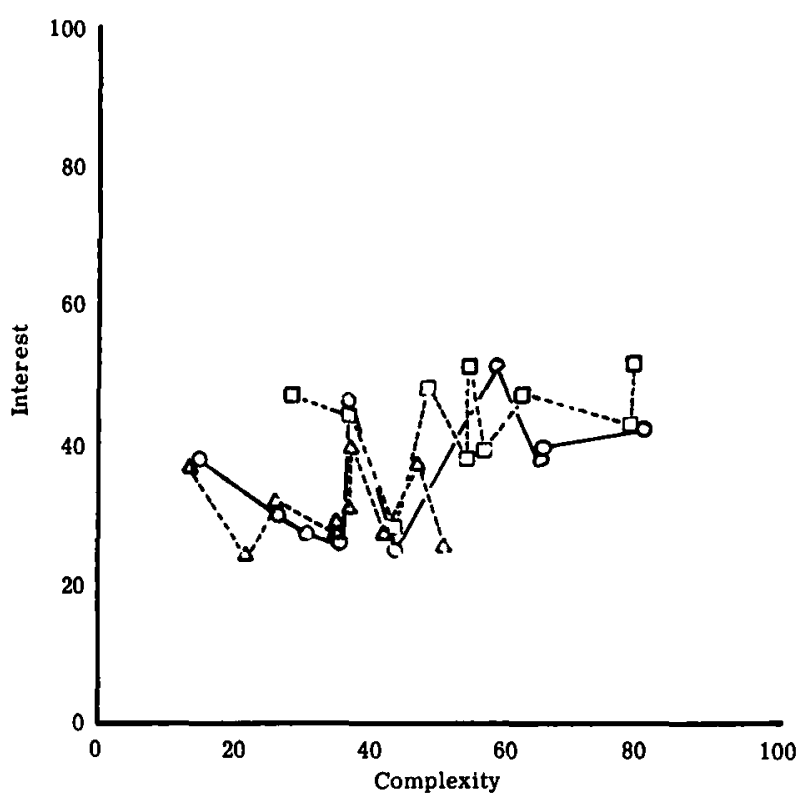

Figure 6. Complexity and "interestingness."

Each group rated all three sets. One group rated the low set, then the high, and then the full range set. Another group rated the high, then the low, and then the full range set. The third group rated the full set first and was then split, with half of the S's rating the low complexity set first and the other half, the high complexity set first. This arrangement comes pretty close to counterbalancing order of presentation. Tests for order effect revealed that there was no systematic order effect, and order was therefore ignored in further analysis.

FIGURE 5 is a plot of the results of the three sets of ratings. It is apparent that when subjects rate a restricted range of stimuli, they tend to use more of the range than they do when rating a wider range. Thus the middle range stimuli were rated as more complex when in a set of low complexity and less complex when in a set of higher complexity.

FIGURE 6 is a plot of the second result of this study. In addition to the complexity ratings, the subjects were asked to rate each pattern for "interestingness." Subjects showed very little consistency in their ratings of this variable. What little there is shows the more complex patterns rated as being more interesting. Since earlier subjects had expressed a liking for the simpler patterns, the two variables, liking and "interestingness," do not appear to be closely related, and what relationship there is appears to be inverse.

Summary of Tartan Studies. This set of materials was selected because it offered a physical dimension, number of squares, with a degree of face validity as a complexity dimension. The physical scale and a simple psychological rating scale showed good agreement.

The relation of complexity and liking was a simple monotonic one, with subjects expressing the greater liking for the simpler patterns. Ratings of "interestingness" and ratings of liking were essentially unrelated.

Comparison of a simple scaling of liking and results from a more complex 
Shepard-Kruskal scaling technique yielded no advantage of the more complex technique, either in terms of the establishment of more than one dimension of liking, or in terms of individual difference analysis.

\section{State Set Designs $\S$}

The design of stage settings is subject to classification with respect to style, and the various styles differ in complexity as one of the descriptive dimensions. In conjunction with a doctoral dissertation on differences in audience reaction to styles of stage setting, a group of 30 photographs of drawings of stage sets became available.

This group of 30 photographs was subjected to two kinds of preliminary appraisal. They were submitted to a panel of scholars in drama for quantitative evaluation of the extent to which each represented any of several styles. They were also rated by a small group of subjects for complexity and preference, using a simple rating procedure. On the basis of these two preliminary procedures, a set of 12 drawings was selected for use in the dissertation. The criterion of choice was primarily that the panel of scholars be in agreement on the style designations.

The settings selected represented three styles, romantic, classical or formal, and baroque. The romantic settings were: one by Chaperon for Faust, one by Adolph Appia for Little Eyolf, one by Karl Fichot for Parsifal, and one by P. L. Ciceri for Ali Baba. The formal or classical settings were: one from Karl Czeschka for King Lear, one from Norman Bel Geddes for Hamlet, one from T. C. Pillartz for Oedipus, and one from Adolph Appia for Orpheus. The baroque settings included one from Fabrizio Galliari, one from Ferdinando Bibiena, and two from Carlo Bibiena. The plays for which they were designed were not designated in the source.

The 12 stage set drawings were then rated four different times by a group of subjects consisting of nine undergraduate and seven graduate students. They were rated for complexity and preference on a simple 7-point scale and rated for both variables, again using the Shepard-Kruskal technique.

The results of the simple rating are shown in FIGURE 7 . The three styles are distinguished in terms of complexity almost without overlap. The four baroque settings are rated as much more complex than the others. The four classicalformal settings are rated on the simple end of the scale. The romantic are in the middle except for one setting that is classed with the formal in terms of complexity.

These subjects express a preference for the baroque settings. The classicalformal are least preferred, but the styles are not differentiated in terms of preference to nearly the extent they are in terms of complexity. Thus, the realtionship between complexity and preference is a monotonically increasing preference for settings of increasing complexity. It might be noted that this is the reverse of the relationship between complexity and preference noted in the studies of tartan patterns.

The Shepard-Kruskal ratings were extensively analyzed, using up to six dimensions in both the analyses of individual data and in the analysis of the group data. Again, the efforts to rationalize the individual data proved utterly fruitless. The analysis of group data was equally frustrating. When the Shepard-

$\S$ This study was done in conjunction with a doctoral dissertation project of Warren Pickett in the Department of Speech and Drama at the University of Michigan. The dissertation, tentatively titled An Experiment in Audience Response to Set Design Style, is to be completed in the Fall term, 1969. 


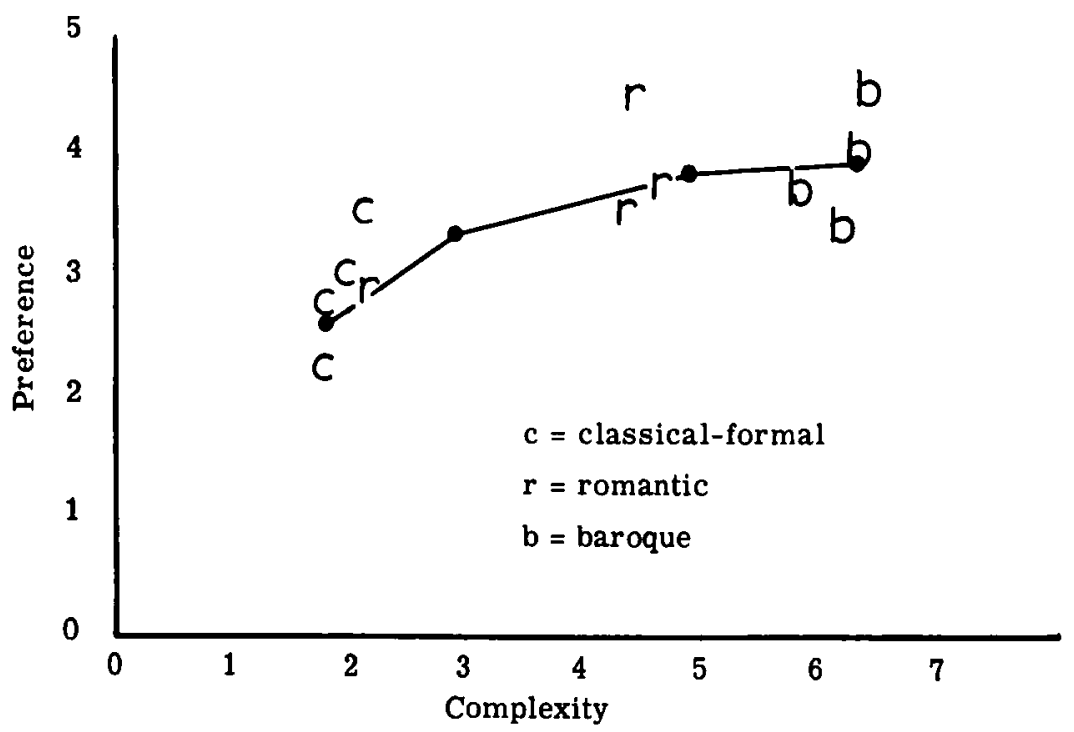

FIGURE 7. Complexity and preference in stage set designs (7-point scales).

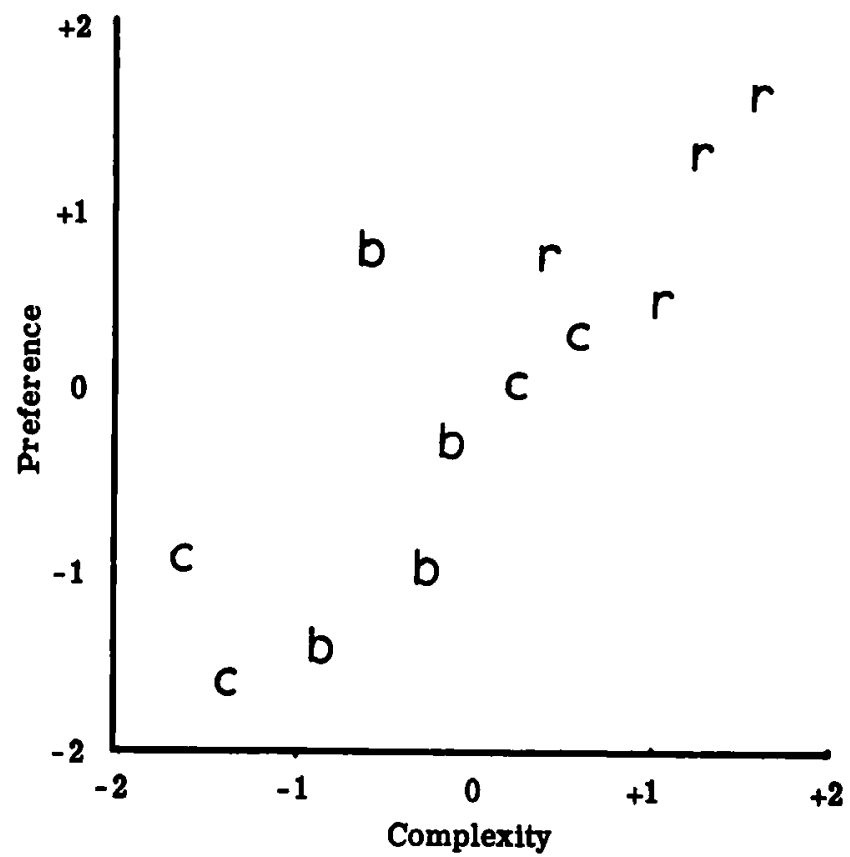

Figure 8. Complexity and preference in stage set designs (Shepard-Kruskal scales). 
Kruskal analyses of complexity were compared with the results of the 7-point scale, the only systematic relationship that emerged was a $\mathbf{U}$-shaped relationship with one of the Shepard-Kruskal dimensions. Sets rated both high and low in complexity on the 7-point scale were at one end of the Shepard-Kruskal dimension, and those in the middle were on the other end.

With six dimensions of complexity and six of preference available, 30 possible pairs can be matched. FIGURE 8 is a plot of the one pairing that showed a systematic relationship. This plot is so linear that one would think that the two dimensions were measurements of the same variable. The other possibility is that this relationship is a chance one drawn from a set of 30 .

The methodological conclusion drawn from these efforts is that the simple rating scale functions as well or better than the more complex multidimensional one, since the latter added no information concerning either variable or the relation between the two.

\section{Complexity, Preference, and Experience with Graphics}

Theories that relate preference to stimulus complexity generally agree in predicting that a subject will prefer a stimulus of moderate complexity rather than an extremely simple or an extremely complex stimulus. The theory stated earlier predicts that with increased experience with a class of stimuli, the preferred stimulus should tend to be chosen from a point closer to the more complex end of the scale.

This study is an attempt to verify the existence of an optimal complexity level in the middle range of a scale of complexity and to determine if the optimal point is higher for subjects with more experience with the range of stimuli. The basic design was to choose a set of graphics that are highly representational in character and that were produced by well-known artists. These were then rated for complexity and preference by a group of law students who were naive with respect to graphic prints, a group of art history students, and a group of graduate students in art. The latter two groups could be expected to recognize many, if not all, of the graphics and to be able to identify the artist in each case.

The first step in the process was to choose 30 prints of graphic art by seven recognized artists. All of the prints were black and white, were the same size $\left(4.5^{\prime \prime} \times 5^{\prime \prime}\right)$ mounted on slightly larger white cardboards and covered with clear acetate for protection.

In the first phase of the study, this set of 30 prints was given to 40 subjects, individually. The group of subjects was equally divided between students in law school and students in either art or art history. They were asked to look through the entire set and then to assign a whole number rating of complexity from 1 to 7 .

Mean ratings for the 30 prints were then used as a basis for choosing a set of 15 prints for the second phase of the study. Five prints each were chosen from the high, mid-, and low portions of the range of ratings, with the additional prescription that the standard deviations of the ratings should be minimal.

In the second phase of the study, 60 subjects forming three groups of 20 subjects each were used. A "naive" group consisted of 20 law students with little experience with graphic materials of the kind used in the study. Few of these subjects were able to recognize any of the prints or identify any of the artists. An "art" group was composed of 20 senior or graduate students in art. An "art history" group was composed of 20 graduate students majoring in art history.

ๆ This study was planned and executed by Kathleen Sinclair and submitted as an Honors Thesis entitled Optimal Complexity and Aesthetic Preference, University of Michigan, 1967. 
That the "art" and "art history" groups had had considerably more experience with the stimulus materials is demonstrated by the fact that all of the members of both groups recognized all of the artists and most of the prints used in the study. The list of prints ordered from most to least complex in terms of the mean complexity score obtained in the first phase of the study is contained in TABLE 2.

The complexity ratings are reasonably stable. The correlation (rho) between

TABLE 2

List of Graphic Prints*

\begin{tabular}{|c|c|}
\hline $\begin{array}{c}\text { Mean } \\
\text { Complexity } \\
\text { Rating }\end{array}$ & Artist and Title of Print \\
\hline $\begin{array}{l}6.4 \\
6.2 \\
5.9 \\
5.6 \\
4.3 \\
4.0 \\
3.95 \\
3.9 \\
3.7 \\
3.6 \\
2.7 \\
2.6 \\
2.4 \\
1.5 \\
1.4\end{array}$ & $\begin{array}{l}\text { Durer, "The Men's Bath" } \\
\text { Durer, "The Sea-Monster," } 1500 \\
\text { Breughel, "The Donkey at School," } 1556 \\
\text { Durer, "Les Trois Graces," } 1491 \\
\text { Durer, "Portrait of the Artist's Mother," } 1514 \\
\text { Chagall, "David Gives Vent to his Grief," } 1931 \\
\text { Toulouse-Lautrec, "La Goulue au Moulin Rouge," } 1891 \\
\text { Goya, "Self Destruction," } 1800 \\
\text { Toulouse-Lautrec, "La Goulue avec une Tanseur," } 1891 \\
\text { Picasso, "The Ball," } 1904 \\
\text { Matisse, "Portrait of Prof. T. Whitmore," } 1937 \\
\text { Chagall, "The Musician," } 1919 \\
\text { Chagall, "My Mother," } 1919 \\
\text { Matisse, "The Princess N" } \\
\text { Matisse, "Henri de Montherlant," } 1937\end{array}$ \\
\hline
\end{tabular}

- The prints that were rated by subjects for complexity and preference in the second phase of the study are arranged in order of the mean complexity values obtained in the first phase.

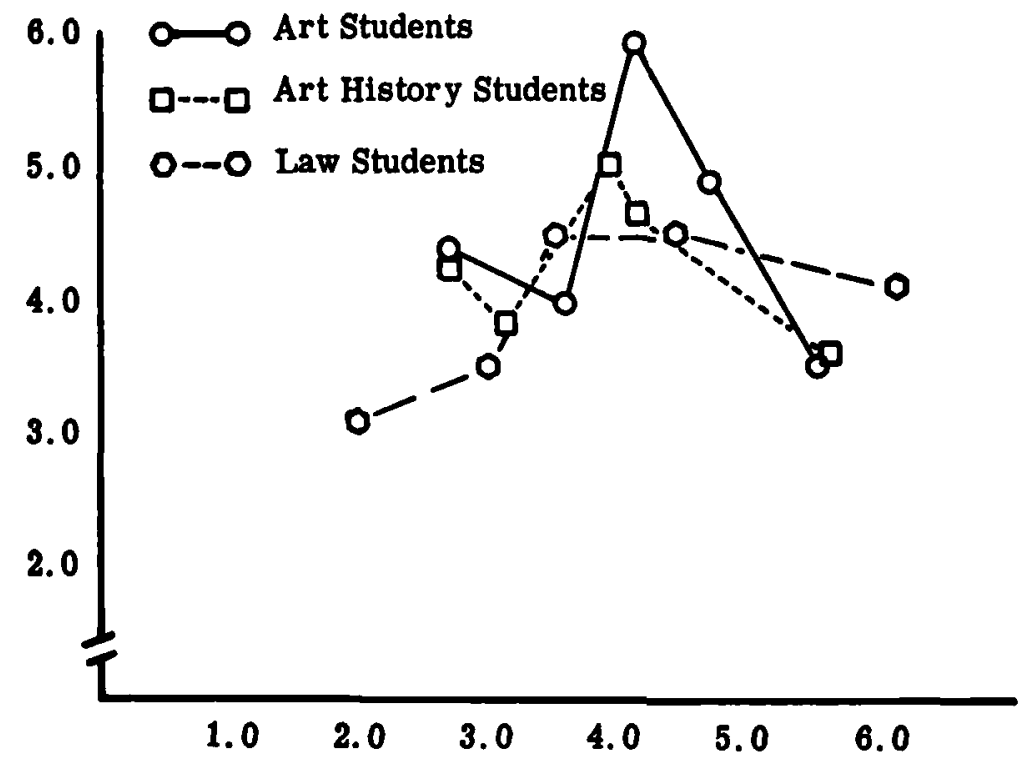

Figure 9. Optimal complexity and experience. 
the ratings in two phases is $\mathbf{9 4}$. The correlations among the complexity ratings given by the three groups are $.93, .94$, and .94 .

FIGURE 9 was achieved by ordering the 15 complexity values and grouping them in sets of three. Means were obtained for each set of three, and the mean preference value for each set was then obtained. The curves in FIGURE 9 are obviously different, and the differences are statistically significant. The curve for the naive subjects is relative flat and they tend to use most of the range of complexity. However, the flatness of the curve indicates that they do not show very consistent preferences except that there is a general preference for the more complex end of the scale. The curves for the more sophisticated subjects are constricted in range on the complexity scale. They tend to see the graphics in a context that makes them more nearly alike in complexity. Both curves show major preference for the middle range of complexity values, an optimum in the middle of the range.

The location of the optimum as a function of the amount of experience is in the direction predicted by the theory. The art history students have considerably less experience with graphics than do the graduate students in art. The arts students show an optimum that is to the right of that for the art history students. However, this apparent difference is not sufficiently great to accept as evidence. A clear demonstration of the movement of the optimum up the complexity scale with increased experience is yet to be demonstrated unequivocally with these materials.

Modern Art. Our most extensive work on measurement techniques and methodology was devoted to the development of a set of materials representing modern abstract art. A large set of stimulus materials was prepared, and from this set, 42 abstract paintings were selected. These were all in color and were abstract in the sense of being nonrepresentational and generally devoid of symbolic material. TABLE 3 contains a list of the paintings used in the preliminary study. The objective was to develop a standardized set of stimulus materials that would then be available for use in studies of complexity and preference, especially studies involving extensive exposure and possible changes in preference with exposure.

The tasks given to subjects involved making four ratings of the paintings. They were to rate complexity and preference on seven-point scales. Then they were to perform the Shepard-Kruskal scaling task, rating differences in complexity or preference between pairs on a scale of 1 to 10 . With 42 stimulus items, the task of rating all possible pairs twice, once for complexity and once for preference, proved to be almost beyond the patience of willing subjects. They complained about the difficulty of the task and the growing unpleasantness. Fifteen subjects agreed to perform the scaling task, and only seven completed the entire sequence of judgments. Each of these seven subjects was then interviewed at length concerning the bases of choices. This interview provided a lengthy protocol against which to compare the scales derived.

The abstract paintings are rich in complexity. They vary in color, technique, degree of homogeneity of line and shape, number of transitions from one color or brightness to another, suggestiveness of meaningful material, and so on. It was felt that ratings of complexity and preference with such materials might well be multidimensional. If they did prove to be multidimensional, the Shepard-Kruskal analysis should reveal dimensions that could be matched against the verbal protocol for each subject.

The general procedure in pursuing this objective was to obtain Shepard-Kruskal analyses from the computer in a number of degrees of detail. Thus, for each subject, the computer generated ten sets of solutions for one dimension through ten 
TAale 3

Modern Abstract Paintings

\begin{tabular}{|c|c|c|c|c|}
\hline $\begin{array}{c}\text { Set } \\
\text { Number }\end{array}$ & $\begin{array}{l}\text { 7-point } \\
\text { Complexity } \\
\text { Rating }\end{array}$ & Painter & Painting & Year \\
\hline \multirow[t]{3}{*}{1} & 5.6 & Jackson Pollack & \#1 & 1949 \\
\hline & 4.5 & Jo Schreiter & Execution of the hope & 1957 \\
\hline & 4.1 & Singier & Les Amoureux et la Plage & 1954 \\
\hline \multirow[t]{2}{*}{ G } & 3.2 & Sugai' & Yamato & 1956 \\
\hline & 3.5 & Tobey & Tatouage & 1958 \\
\hline 2 & 6.1 & Singier & L'Eté & 1945 \\
\hline 3 & 3.4 & Hofmann & Pompei & 1959 \\
\hline 4 & 2.4 & Sugai' & Yayoi & 1958 \\
\hline \multirow[t]{2}{*}{5} & 5.8 & Willi Baumeister & Animated landscape & 1946 \\
\hline & 2.5 & Stamos & Avignon II & 1958 \\
\hline \multirow[t]{2}{*}{6} & 1.4 & Piet Mondriaan & Composition & 1927 \\
\hline & 4.5 & Max Ackermann & Jubilation & 1954 \\
\hline $\mathbf{F}$ & 3.4 & Piet Mondriaan & Composition & 1913 \\
\hline 7 & 5.5 & Corneille & Tropical splendour & 1958 \\
\hline 8 & 5.3 & Wassily Kandinsky & Pink Composition & \\
\hline \multirow[t]{3}{*}{9} & 2.3 & Soulages & Peinture & 1957 \\
\hline & 4.9 & Corneille & Landscape of America & 1958 \\
\hline & 2.4 & Dubuffet & Table & 1957 \\
\hline $\mathbf{E}$ & 4.7 & Vieira Da Silva & Red Interior & 1951 \\
\hline \multirow[t]{4}{*}{10} & 5.5 & Marca-Relli & Summer noon & 1956 \\
\hline & 4.4 & Bram Van Velde & $\begin{array}{l}\text { Composition } \\
\text { Collection Marie Cuttoli }\end{array}$ & 1957 \\
\hline & 4.9 & Bram Van Velde & Composition & 1957 \\
\hline & 4.0 & Lyonel Feininger & Month of R. Rega III & \\
\hline 11 & 6.4 & Goodnough & Summer & $1959-60$ \\
\hline 12 & 2.1 & Stamos & Persian Bride & 1958 \\
\hline 13 & 4.3 & Le Moal & Ocean & 1959 \\
\hline \multirow[t]{2}{*}{ D } & 3.3 & Singier & Soleil et sable & 1956 \\
\hline & 3.3 & Sugai' & Oni & 1958 \\
\hline 14 & 5.1 & Prassinos & Le Clown & 1960 \\
\hline 15 & 1.5 & Mark Rothko & Number 10 & 1950 \\
\hline C & 4.5 & Schneider & Peinture $65 \mathrm{~B}$ & 1954 \\
\hline $\mathbf{B}$ & 5.8 & Dubuffet & Garden Bleche Grignotte & 1956 \\
\hline 16 & 3.8 & Poliakoff & Oil & 1953 \\
\hline 17 & 2.8 & Sugai & Sugata & 1958 \\
\hline 18 & 3.5 & Schneider & Peinture 95 B & 1955 \\
\hline \multirow[t]{3}{*}{19} & 4.4 & Tobey & Intersection & 1954 \\
\hline & 4.1 & Tobey & Pacific Circle & 1956 \\
\hline & 3.5 & Soulages & Peinture & 1958 \\
\hline \multirow[t]{2}{*}{20} & 3.0 & Pierre Soulages & Composition & \\
\hline & 4.3 & Tobey & Summer & 1957 \\
\hline 21 & 3.9 & Goetz & Peinture & 1954 \\
\hline A & 2.8 & Tobey & Yellow Harvest & 1956 \\
\hline
\end{tabular}

dimensions, i.e., 55 dimensions for complexity and 55 for preference for each of 7 subjects.

Analyses of the data for an individual subject tended to take the following form. The one-dimensional solutions and the two dimensions of the two-dimensional solutions for complexity and preference were plotted for every individual, and many pairings of higher level were plotted. The resulting plots varied in the extent of apparent relations, but examination of a large number of such plots revealed nothing that was meaningfully different from the later analysis of the group data. The second step was to use the Shepard-Kruskal dimensions in pairs to arrange the paintings in two-dimensional arrays. A room wall was utilized for 
this purpose. A vertical scale was fastened to the wall from floor to ceiling, and a horizontal scale was fastened to the wall from left to right. Then, for example, the two dimensional Shepard-Kruskal solution for an individual subject would be used to place each of the 42 paintings on the wall in accordance with its assigned values from the analysis. If the scales were complexity scales, then the array would be examined to see whether there was any way of making sense of the dimensions in terms of complexity. If the array represented preference, then they
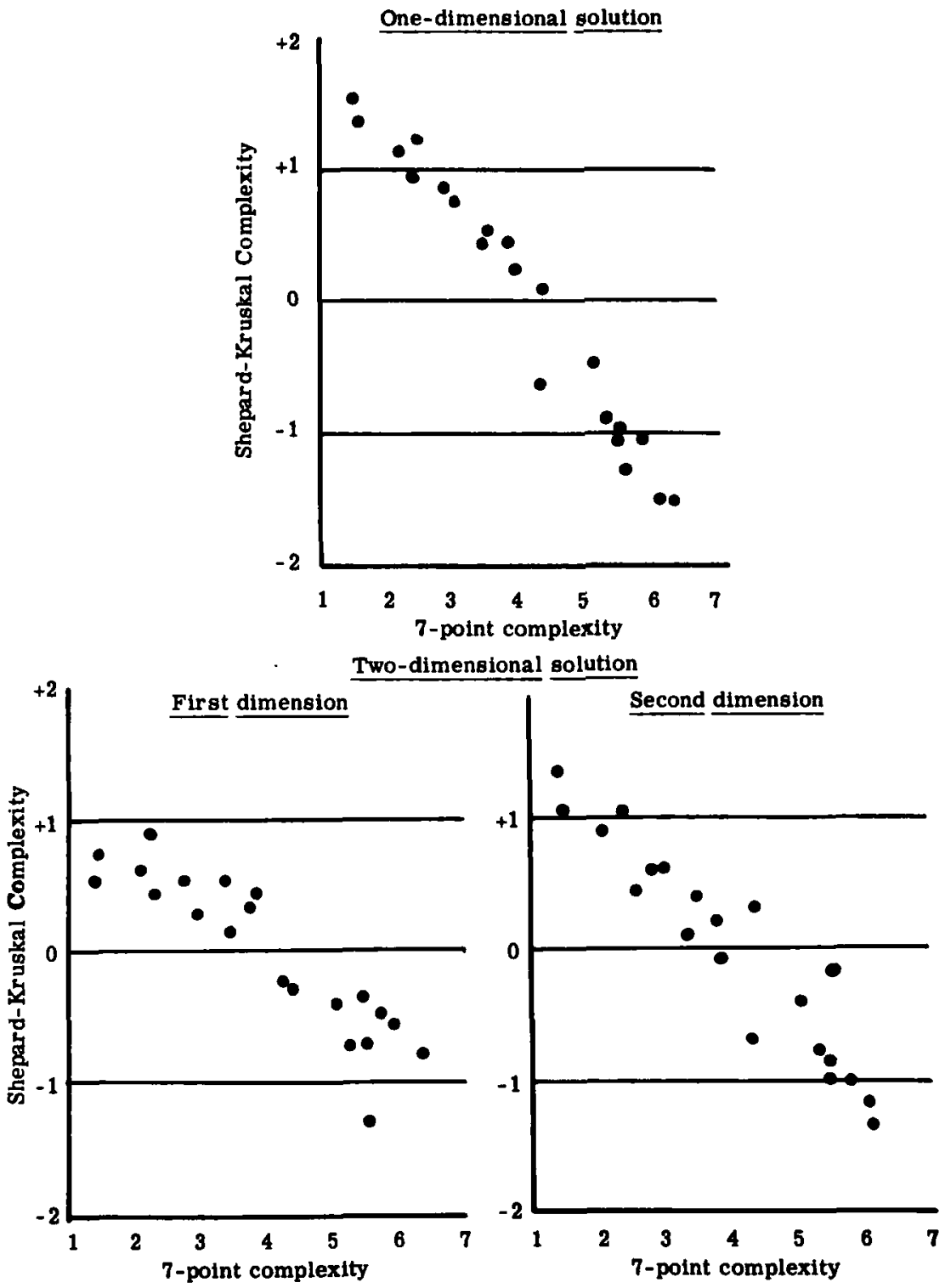

FigURE 10. Comparison of 7-point and Shepard-Kruskal scales of complexity. 


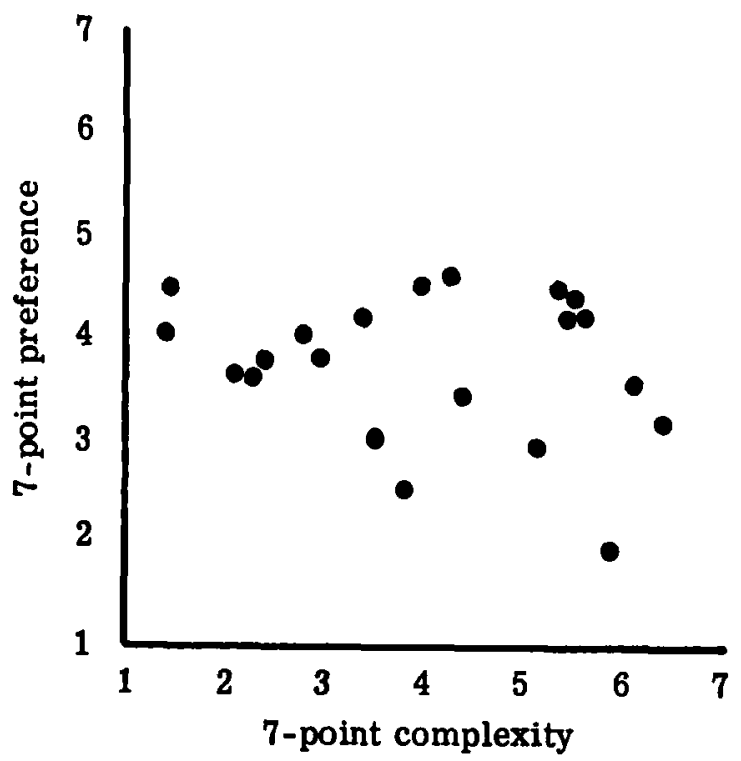

Figure 11. Complexity versus preference for modern paintings (7-point scale data).

were examined to ascertain whether the basis of preference along each of the dimensions could be determined. These examinations were aided by the protocols taken from the subjects in an effort to determine why a given painting should occupy that particular position in the array. Several experimenters spent a great many hours at this task. Many arrays were examined by as many as seven or eight people. Many arrays were photographed in color and examined later in an effort to establish meaningfulness. Although it was not done systematically, a number of arrays was examined by the subjects who had provided the original data. The net outcome was the conclusion that multidimensional analyses of these data did not yield more than one dimension of either complexity or preference in the data of individual subjects.

For a variety of reasons, it was decided that working with 42 stimuli was unnecessarily cumbersome. The mean 7 -point complexity values for the 15 subjects was then used to reduce the set of 42 paintings to a set of 21 experimental paintings and a practice set of six. TABLE 3 contains the mean complexity values. It also contains the code number assigned to the 21 paintings of the experimental set, and the letters $A$ through $G$ for the practice set that could be used in instructing future subjects.

This small set was then rated by 15 subjects, on both 7-point and ShepardKruskal scales in an effort to determine the extent of agreement of the two scaling techniques and whether any advantage accrued from the use of the more complicated Shepard-Kruskal procedure.

FIGURE 10 shows a comparison of the 7-point ratings of complexity compared to the one-dimensional and both dimensions of the two-dimensional ShepardKruskal solutions. In all three cases there is good agreement in these grouped data. If a stimulus is rated complex on one scale, it is likely to be rated as complex by use of the other method. The two dimensions of the two-dimensional 
Shepard-Kruskal solution both show good agreement, suggesting that they are highly correlated dimensions within the Shepard-Kruskal analysis.

FIGURE 11 is a plot of the 7-point complexity and preference values. Almost no trend is apparent in the data beyond a minor tendency for mean preference for some of the more complex stimuli to be somewhat low. Figure 12 shows a comparison of the Shepard-Kruskal one dimensional solutions for complexity and preference. Again, no reliable trend is apparent.

FIGURE 13 contains the plots of 7-point complexity ratings and three ShepardKruskal dimensions of preference. None shows relationships.

Two conclusions seem justified on the basis of these studies. The first is that it seems probably that when one asks a subject to rate a set of abstract paintings for complexity or preference, the subject does just that. It is a simple pair of dimensions from the standpoint of the subject and it is thus not composed of contributing sets of underlying dimensions. The second conclusion is that in these data, no systematic relationship between complexity and preference could be demonstrated.

A possible explanation for the lack of relationship between complexity and preference in these data might lie in the experience factor. The theory of complexity predicts that there will be changes in complexity and preference with experience. It could be that such changes were occurring rapidly enough that the mean judgments represented valid judgments from different points in time that were not coordinate because of the order or presentation and the amount of experience provided in the experimental situation.

Based on this possibility, an effort was made to introduce a simple control of experience within the setting and collect a new set of data with a larger number

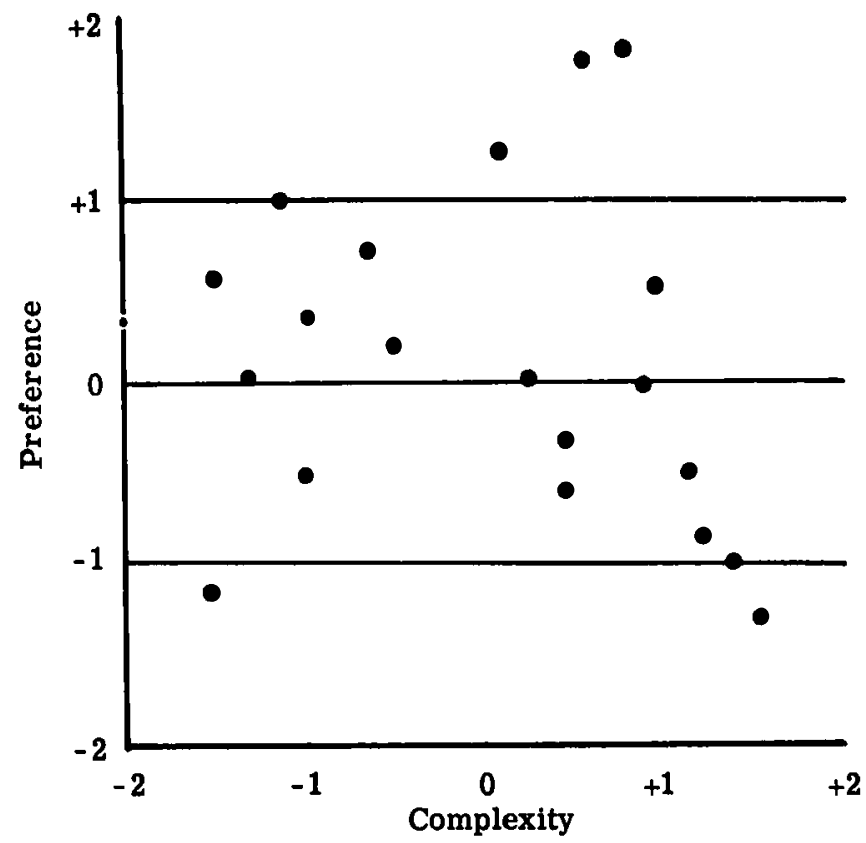

Figure 12. Complexity versus preference for modern paintings (Shepard-Kruskal scale data). 
Walker: Complexity and Preference
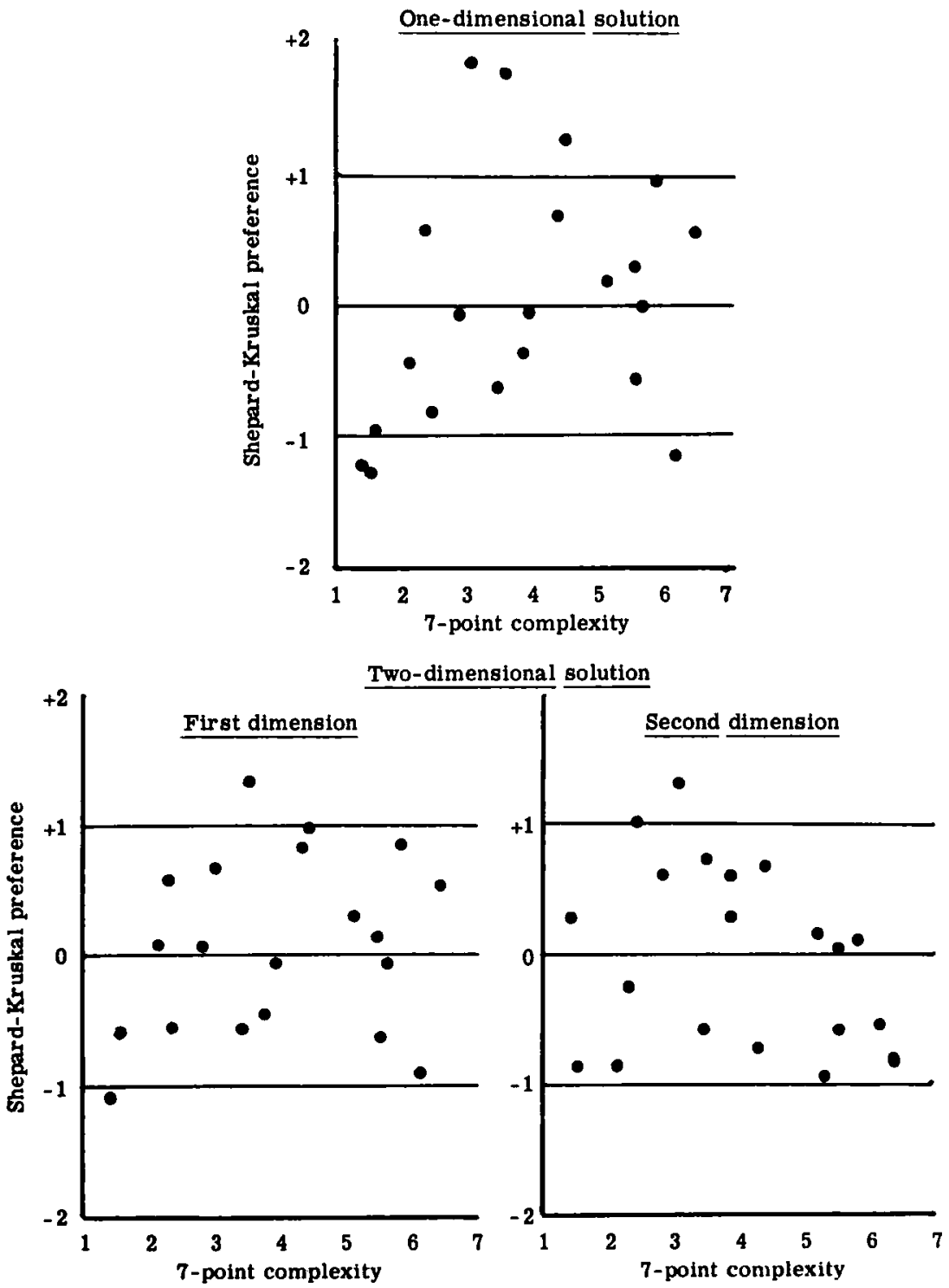

FIgURE 13. Complexity versus preference for modern paintings (7-point scales of complexity versus Shepard-Kruskal preference solutions).

of subjects. The simple 7-point scaling procedure was used. The demonstration set of six paintings was used to explain the procedure. The 40 subjects were then asked to rate each of the 21 pictures for complexity and preference.

The major results in terms of complexity and preference is plotted in FIGURE 14. There is a definite relation between the two, with preference being expressed for the more complex of the paintings. 
Summary of Results from Human Studies of Complexity and Preference

The major effort in this area was to develop several sets of visual stimulus materials for use in future research. The research has produced four sets, each of which yields reliable ratings of complexity and preference.

The methodological conclusions are simple and somewhat disappointing. Multidimensional analysis of ratings of complexity and preference does not seem fruitful. The simplest rating tasks, in which the subject is asked to assign an absolute rating from 1 to 7 , from 1 to 10 , or from 1 to 100 , seem to yield results that are as functional for future research as the more complex scaling techniques. The Shepard-Kruskal technique imposes a very difficult task on the subject and requires that the subject be exposed to the stimuli a number of times and for considerable periods. It seems likely that the complexity value and preference for the stimuli are undergoing extensive change during the process of rating.

The search for an optimum preference value along the complexity dimension yielded rather varied results. The "best"" result with each of the four sets of stimulus materials is plotted in FIGURE 15. Subjects express maximum preference for the simplest of the tartan patterns. They show a maximum preference for black and white graphics in the middle range of complexity. Maximum preference is expressed for the most complex of the stage settings and the abstract paintings.

The effect of experience on complexity and preference was explored systematically in only one study. In that one, the more experienced art students used a more restricted range of complexity ratings and a wider range of preference ratings than did the more naive subjects. Optimal preference for middle values of complexity was evident in this study, but a significant increase in the complexity of optimal preference with experience was not demonstrated.

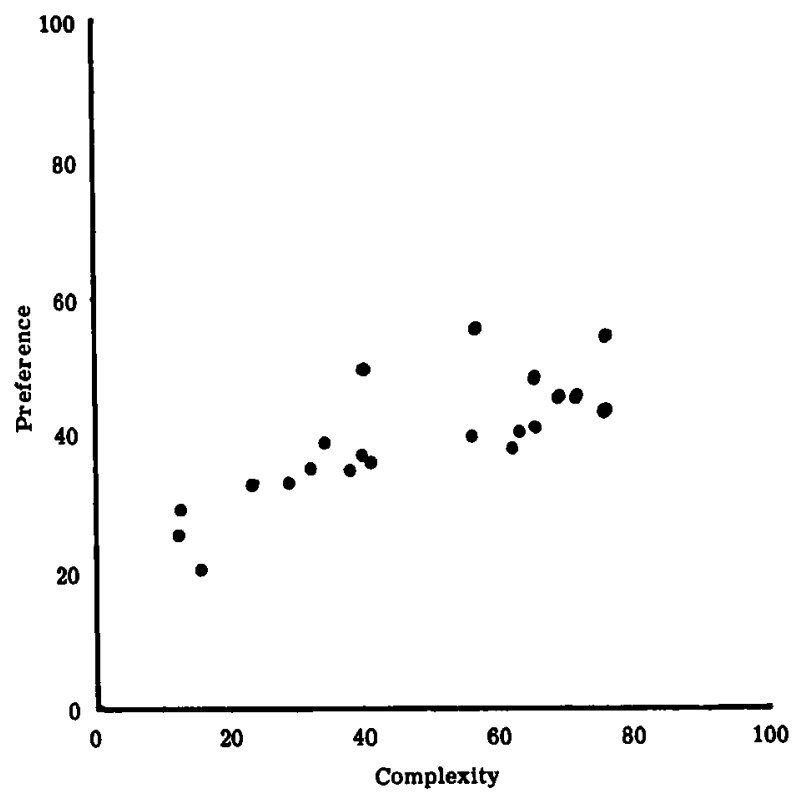

Flgure 14. Complexity versus preference for modern paintings. 
TARTAN PATTERNS

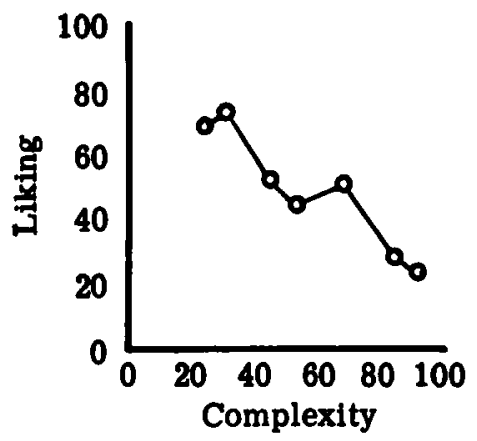

GRAPHIC PRINTS

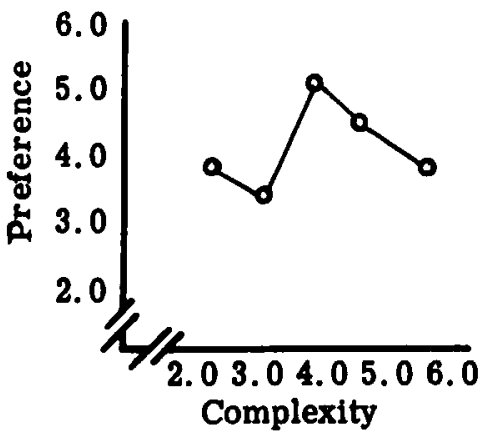

STAGE SET DRAWINGS

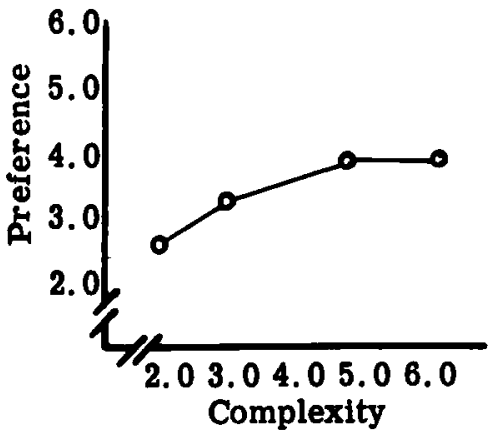

ABSTRACT PAINTINGS

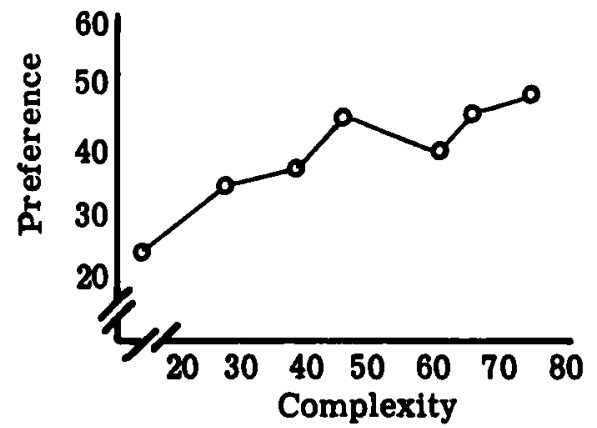

FIGURE 15. Summary of complexity and preference relations.

\section{Stimulus Complexity and Preference in Animals}

It is easy to ask a human subject how complex he thinks a given stimulus is and then to ask him to rate his preference for it. It is more difficult to have an animal try to distinguish between the two questions. If one presents an array of stimuli to an animal and if the animal approaches one element of that array, it is reasonable to assume that he prefers that stimulus to the others. If the array is composed of stimuli that have a dimension of complexity that is obvious to the experimenter, then one can infer that the animal has expressed a preference for the chosen stimulus on the basis of its relative complexity. However, the inference is not a strong one. If, in the pattern of successive choices, the animal behaves in a manner that is orderly along this complexity dimension, the inference that it is the complexity of the stimulus to which he is responding seems strengthened.

The lack of independence of the judgments of complexity and preference is the major disadvantage of working with animals on this problem. The advantage lies 
in the assumed relative simplicity of the animal and the degree of control that the experimenter can exercise. Animal research on complexity and preference was undertaken in the light of the obvious advantages and disadvantages.

Two-dimensional versus three-dimensional complexity in the rat. The first study of this series ${ }^{8}$ was an effort to explore the question whether repeated experience with two sets of stimuli (two dimensional and three-dimensional) would produce a progressively changing preference toward more complex stimuli. One stimulus set consisted of simple wall patterns composed of (1) a uniform grey, (2) a set of horizontal stripes, (3) a set of vertical stripes, and (4) a checkered pattern. The three-dimensional patterns consisted of (1) an empty compartment, (2) a compartment with one baffle, (3) a compartment with two baffles, and (4) a compartment with a complex set of baffles greater than two. It was assumed that these two sets of stimuli represented two dimensions of complexity, both of which were anchored on the empty grey compartment.

A group of 16 animals was run in each maze. They were run individually and left in the maze for a period of 30 minutes on each of five successive days. The sequence of choices and time spent in each compartment were recorded.

The compartment in which an animal spends more time than any other can be designated as his preferred compartment for that day. TABLE 4 is a tabulation of preferred compartment for each day for each group. The stimuli are ordered in the Table in the a priori order of complexity with respect to which they were designed.||

TABLE 4

Preferred Compartments by Days

\begin{tabular}{|c|c|c|c|c|}
\hline \multirow[b]{2}{*}{ Days } & \multicolumn{4}{|c|}{ Wall Patterns } \\
\hline & Grey & $\begin{array}{l}\text { Horizontal } \\
\text { Stripes }\end{array}$ & $\begin{array}{l}\text { Vertical } \\
\text { Stripes }\end{array}$ & Checks \\
\hline $\begin{array}{l}1 \\
2 \\
3 \\
4 \\
5\end{array}$ & $\begin{array}{c}16 \\
16 \\
14 \\
13 \\
7.5\end{array}$ & $\begin{array}{l}\mathbf{0} \\
0 \\
1 \\
2 \\
1\end{array}$ & $\begin{array}{l}0 \\
0 \\
1 \\
1 \\
3.5\end{array}$ & $\begin{array}{l}0 \\
0 \\
0 \\
0 \\
4\end{array}$ \\
\hline 5 & \multicolumn{4}{|c|}{ Baffle Patterns } \\
\hline Days & Grey & One Baffle & Two Baffles & Three-plus baffles \\
\hline 1 & 0 & 6.5 & 4.5 & 5 \\
\hline 2 & 1 & 2 & 0 & 13 \\
\hline 3 & 0 & 0 & 1 & 15 \\
\hline 4 & 0 & 1 & 0 & 15 \\
\hline 5 & $\mathbf{0}$ & 1 & 0.5 & 14.5 \\
\hline
\end{tabular}

In the wall pattern maze, the simplest compartment with grey walls is preferred by every animal for the first two days. On subsequent days, increasing number of animals express preference for increasingly complex wall patterns. In the baffle maze, preference is expressed about equally for the three more complex patterns on the first day. By the second day the animals are almost universally expressing a preference for the most complex of the quadrants.

Some of the problems of interpretation of these results may be seen more clearly if the data are plotted on a hypothetical scale of stimulus complexity. This has been done in FIGURE 16. Each number represents the mean preference for the

II The analyses presented here differ slightly from those presented in reference 8 . 


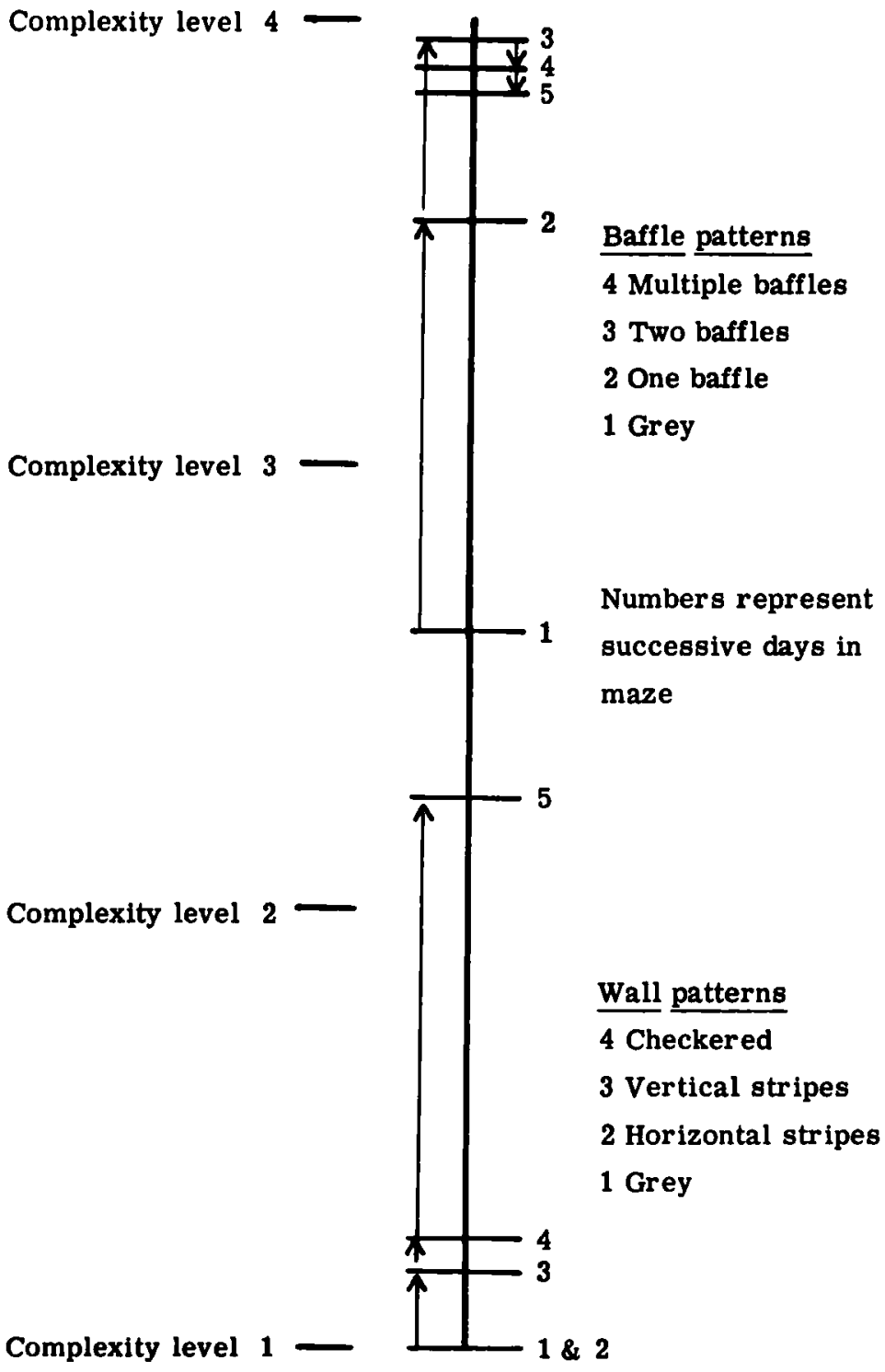

Figure 16. Stimulus complexity scale.

day (number of animals $\times$ the arbitrary complexity value/N). The animals exposed to the wall patterns show a predominant preference for the grey compartment on the first two days, then move to a slight preference for more complex patterns on the third and fourth days, and make a significant shift upward only on the fifth day. The animals with the baffle patterns show a preference midway on the scale on the first day, and move very close to the top by the second.

The scale in FIGURE 16 is dependent on the arbitrary assignment of complexity 
scale values of " 1 " for the grey compartment in each case, a value of " 2 " for the next most complex, and a value of " 3 " and the " 4 " for the next two. These values assume equal metric values for the horizontal stripes and for the one baffie, equal value for vertical stripes and two baffles, and equal weights for the checkered pattern and the multiple baffles. Without an appeal to the theory there is no simple way to determine which set of stimuli, the two-dimensional or the threedimensional, is the more complex.

If we take the theory seriously, then we could reason in the following fashion. Since the animals took several days to venture at all into the more complex twodimensional patterns in contrast to the immediate approach to the baffle patterns, then the wall patterns are probably the more complex and should be assigned complexity values greater than those assigned the baffle patterns. However, there is no simple way to determine what scale values to assign to the stimuli.

An alternative is to combine into one maze representatives of the two types of stimuli. To do this, the same maze compartments were redesigned with the following stimulus patterns.**

1. Grey walls, no baffles

2. Grey walls, one baffle

3. Horizontal stripe walls, no baffles

4. Vertical stripe walls, three baffles

The first of these, the grey walls without baffles, seemed to be an obvious choice for the simplest stimulus pattern we could devise. The fourth, the vertical striped pattern with the three baffles was a combination of the two most complex of the two previous sets of stimuli. The other two seemed to be middle values. If one had to guess, on the basis of previous results, one would guess that the one baffle pattern would be the simpler of the two.

Only three rats were used in the study, but each of the three was exposed to the stimuli for continuous sessions of 100 minutes each on six consecutive days. It was hoped that the much longer exposure times would be adequate to permit significant changes in preference.

In order to analyze the data, each session was arbitrarily divided into ten periods of ten minutes each. For each such time block, the quadrant in which the animal spent the majority of his time was designated as the preferred compartment.

TABLE 5 is a tabulation of the number of blocks out of 30 (ten each for three animals) in which each quadrant was preferred, on each of the six days. The data are generally orderly, in spite of the small number of animals. The initial preference is clearly for the simplest of the quadrants, and the final preference clearly for the most complex. The guess that the baffle pattern would be less complex than the horizontal stripes seems supported by the fact that the preference for the horizontal stripes is expressed later in the time sequence.

The data can be analyzed within days as well. This has been done in TABLE 6 . Again, the data seem orderly despite the handicap of the small number of animals and the fact that the preference is changing markedly from day to day, as shown in TABLE 5. Looking down the columns, the preference for the one baffle quadrant declines in the 100-minute period, while the preference for the two more complex patterns increases slightly. Looking across the rows, the theory would state that the pattern one would not expect would be one that declined and then in-

\footnotetext{
* This study was done by Bruce E. Walker and is unpublished. Its title might be Scaling of Two-Dimensional and Three-Dimensional Stimulus Complexity in the Rat.
} 
TABLE 5

Preferred Stimuli by Days

\begin{tabular}{ccccc}
\hline Day & Grey & 1 Baffe & $\begin{array}{c}\text { Horizontal } \\
\text { Stripes }\end{array}$ & $\begin{array}{c}\text { Vertical Stripes } \\
\text { and 3 Baffes }\end{array}$ \\
\hline 1 & 20 & 9 & 0 & 1 \\
2 & 6 & 17 & 7 & 0 \\
3 & 1 & 15 & 14 & 0 \\
4 & 2 & 12 & 12 & 4 \\
5 & 2 & 13 & 5 & 10 \\
6 & 0 & 8 & 7 & 15 \\
\hline
\end{tabular}

TABLE 6

Preferred Stimula ay Successive Ten-Minute Periods

\begin{tabular}{ccccc}
\hline Minutes & Grey & 1 Baffle & $\begin{array}{c}\text { Horizontal } \\
\text { Stripes }\end{array}$ & $\begin{array}{c}\text { Vertical Stripes } \\
\text { and 3 Baffles }\end{array}$ \\
\hline $1-10$ & 2 & 14 & 0 & 2 \\
$11-20$ & 1 & 11 & 4 & 2 \\
$21-30$ & 4 & 9 & 3 & 2 \\
$31-40$ & 3 & 9 & 4 & 2 \\
$41-50$ & 5 & 6 & 5 & 2 \\
$51-60$ & 4 & 6 & 6 & 3 \\
$61-70$ & 2 & 6 & 7 & 5 \\
$71-80$ & 4 & 3 & 5 & 5 \\
$81-90$ & 3 & 5 & 5 & 5 \\
$91-100$ & 3 & 5 & 5 & \\
\hline
\end{tabular}

creased. In only two of the 30 instances is the expectation not met. Thus there is a general tendency for the animals to move from less complex to more complex stimuli during the day.

Analysis of Sequences of Choice. The theory relating complexity and preference indicates that the expressed preference should be for progressively more complex stimuli. In the simplest form of the theory, there should be no regressions. That is, if an animal first prefers the grey compartment and then progresses to a preference for the compartment with one baffle, he should not again express a preference for the plain grey compartment. The prediction does not contain a statement concerning variability or error. It simply states that if we number the quadrants from 1 through 4 , the preference sequence should be $1,2,3,4$, and no sequence such as $1,2,3,2,3,4$ should be observed. Rigid adherence to the complexity sequence seems too much to expect, yet the theory makes no prediction related to the variability in the sequence.

Analysis of sequences has an inherent problem. One way of analyzing the data would be to list a sequence of choices, in this case compartment entries, and then analyze the sequence for sequential dependencies. However, such a sequence of choices would not take the amount of time spent in each compartment into account. An alternative analysis would simply be the total amount of time spent in each compartment. This analysis yields no information on sequences of choices. A compromise analysis is in terms of arbitrary time blocks. In this case, one can use the ten-minute time blocks that were used in the previous analysis. For each time block, the quadrant in which the animal spent the most time can be designated as the preferred stimulus for that time block. Such an analysis loses data on brief visits and differs slightly from analyses in terms of total time spent 
in each quadrant. However, it does have the advantage of representing the sequence of choices made and the time spent in each compartment and, therefore, in the presence of each stimulus.

In this study, if one takes the sequences of designations of preferred stimuli for each animal over the six days, the sequences consist of 60 successive designations of preferred stimulus for each animal. Each sequence can then be analyzed by tabulating the frequency with which each designation of preference is followed by each other designation. The frequencies can then be converted to first-order transitional probabilities. This has been done for each animal in TABLE 7. It has been done for all three animals but for Days 1 and 2, Days 3 and 4, and Days 5 and 6 in pairs in TABLE 8 . TABLE 8 also contains the suminary table of transitional probabilities for all six days for all three animals.

The major individual differences in TABLE 7 are that Animal X rarely entered the most complex quadrant with the vertical stripes and the multiple baffles. Animal $Y$, on the other hand, visited the horizontal striped compartment rather infrequently. If the complexity ordering in the Table is correct, this animal jumped directly from the one-baffle pattern to the most complex. There appears to be no simple way to unfold a scale based on nothing but sequences to order the stimuli for individual animals, but it could be that for Animal Y, the complexity order of the one-baffle and the horizontal stripes is reversed from the order in the Table.

The analysis of transitional probabilities by days in TABLE 8 shows a clear progression given the a priori ordering of the stimulus patterns. The action is relatively confined to the two simpler stimuli on the first two days. It moves to the second and third stimuli on the next two days, and progresses to include the most complex stimulus on the final two days.

While complexity theory does not predict the amount of variability in choice

TABLE 7

Transitional Probabilities of Stimulus Preference by Animal

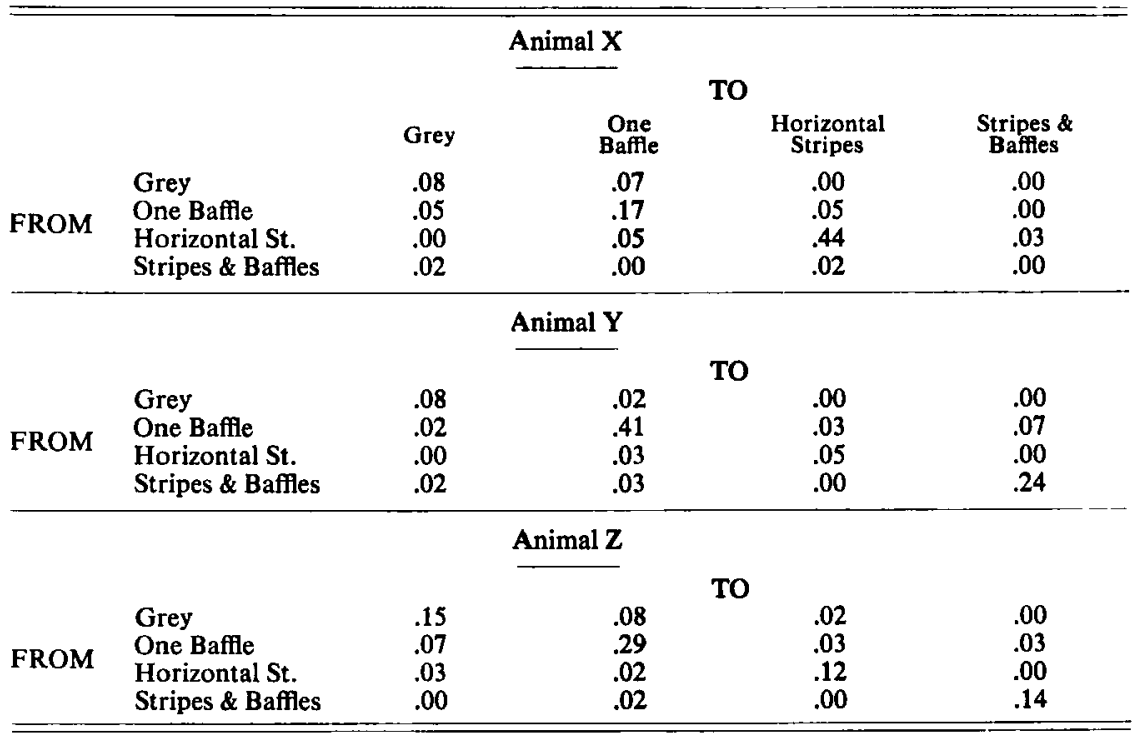


TABle 8

Changing Preferences for Stimulus Complexities Over Time in Terms of Transitional Probabilities

\begin{tabular}{|c|c|c|c|c|c|}
\hline \multirow[b]{3}{*}{ FROM } & \multirow[b]{3}{*}{$\begin{array}{l}\text { Grey } \\
\text { One Baffle } \\
\text { Horizontal St. } \\
\text { Stripes \& Baffles }\end{array}$} & \multicolumn{4}{|c|}{ Days 1 and 2} \\
\hline & & Grey & $\begin{array}{c}\text { One } \\
\text { Baffle }\end{array}$ & $\begin{array}{c}\text { Horizontal } \\
\text { Stripes }\end{array}$ & $\begin{array}{c}\text { Stripes \& } \\
\text { Baffles }\end{array}$ \\
\hline & & $\begin{array}{l}.34 \\
.12 \\
.00 \\
.02\end{array}$ & $\begin{array}{l}.11 \\
.27 \\
.02 \\
.00\end{array}$ & $\begin{array}{l}.00 \\
.04 \\
.09 \\
.00\end{array}$ & $\begin{array}{l}.00 \\
.02 \\
.00 \\
.00\end{array}$ \\
\hline & & & & id 4 & \\
\hline \multirow[t]{2}{*}{ FROM } & $\begin{array}{l}\text { Grey } \\
\text { One Baffle } \\
\text { Horizontal St. } \\
\text { Stripes \& Baffles }\end{array}$ & $\begin{array}{l}.00 \\
.02 \\
.04 \\
.00\end{array}$ & $\begin{array}{l}.04 \\
.37 \\
.02 \\
.00\end{array}$ & $\begin{array}{l}.02 \\
.05 \\
.37 \\
.02\end{array}$ & $\begin{array}{l}.00 \\
.02 \\
.02 \\
.04\end{array}$ \\
\hline & & \multicolumn{4}{|c|}{ Days 4 and 5} \\
\hline \multirow[t]{2}{*}{ FROM } & $\begin{array}{l}\text { Grey } \\
\text { One Baffle } \\
\text { Horizontal St. } \\
\text { Stripes \& Baffles }\end{array}$ & $\begin{array}{l}.00 \\
.02 \\
.00 \\
.02\end{array}$ & $\begin{array}{l}.04 \\
.23 \\
.02 \\
.05\end{array}$ & $\begin{array}{l}.02 \\
.02 \\
.18 \\
.00\end{array}$ & $\begin{array}{l}.00 \\
.09 \\
.00 \\
.34\end{array}$ \\
\hline & & \multicolumn{4}{|c|}{ Days 1 through 6} \\
\hline FROM & $\begin{array}{l}\text { Grey } \\
\text { One Baffle } \\
\text { Horizontal St. } \\
\text { Stripes \& Baffles }\end{array}$ & $\begin{array}{l}.11 \\
.05 \\
.01 \\
.01\end{array}$ & $\begin{array}{l}.06 \\
.29 \\
.03 \\
.02\end{array}$ & $\begin{array}{l}.01 \\
.04 \\
.20 \\
.01\end{array}$ & $\begin{array}{l}.00 \\
.03 \\
.01 \\
.12\end{array}$ \\
\hline
\end{tabular}

sequence one might expect, it does make some predictions concerning a table such as that on the bottom of TABLE 8. If the progression of preferences was ordered absolutely, then all of the probabilities of the table would be along the diagonal except for three transitions, from 1 to 2 , from 2 to 3 , and from 3 to 4 , and there should be no reversals, e.g. from 3 to 2 . It is apparent that the preference sequences are not ordered absolutely.

A weaker prediction would be that the transitional probabilities would depart from a chance distribution. It is obvious, without test, that this prediction is fulfilled. The theory also makes a prediction concerning the character of the dependency. If the animals are responding to the stimuli and are reacting to them as they have been ordered on an $a$ priori basis, then the interaction between adjacent stimuli should be greater than more remote stimuli. The sum of the probabilities in the diagonals adjacent to the main diagonal is .20 . The sum of the more remote interactions (the three in the lower left and the three in the upper right) is .08 . This result would tend to confirm that the animals were indeed responding to the complexity scale and that they were responding to it as it is ordered in the Tables.

Generalized Complexity Satiation. $\$$ Complexity theory predicts that with continued exposure to a stimulus dimension, the organism should show preference for

$\S \S$ This study was carried out by Bruce E. Walker and is unpublished. It might be titled Effect of Prior Exposure to One Stimulus on Preference for Other Stimuli on the Same Dimension. 
stimuli of increasing complexity, and this prediction has been repeatedly verified in the previous studies. If there is generalization of the effects of exposure to one stimulus on a dimension, it should have an influence on the relative preference for stimuli on the same dimension to which the organism has not been exposed.

To test this proposition, a study was designed in the following pattern. Five stimuli were constructed that should represent a dimension of stimulus complexity to the rat. The five patterns were all vertical stripe patterns of black and white and they varied in width. The widths were:

$\begin{array}{lr}\text { Most complex } & 3 / 16 \text {-inch stripes } \\ & 3 / 8 \text {-inch stripes } \\ \text { Middle complexity } & 3 / 4 \text {-inch stripes } \\ \text { Least complex } & 1 \& \text { 1/2-inch stripes } \\ \text { 3-inch stripes }\end{array}$

The basic plan was to vary the amount of prior exposure of groups of animals to the middle stimulus and then to observe the effect of the different durations of exposure on the preferences of the animals when given choices among the other four.

It was felt that the total amount of exposure in the maze should be held constant, in this case at one hour, but that the duration of exposure to the $3 / 4$-inch stripe pattern should be varied. To do this, the four-compartment maze was employed, and all three groups were initially placed in the maze with the walls a uniform grey. A short exposure group was created by leaving the animals in the grey condition for 50 minutes, removing them while the stripe pattern was installed, and then putting them back for ten minutes to complete the hour of exposure. A middle exposure group was created by exposing them to the grey for

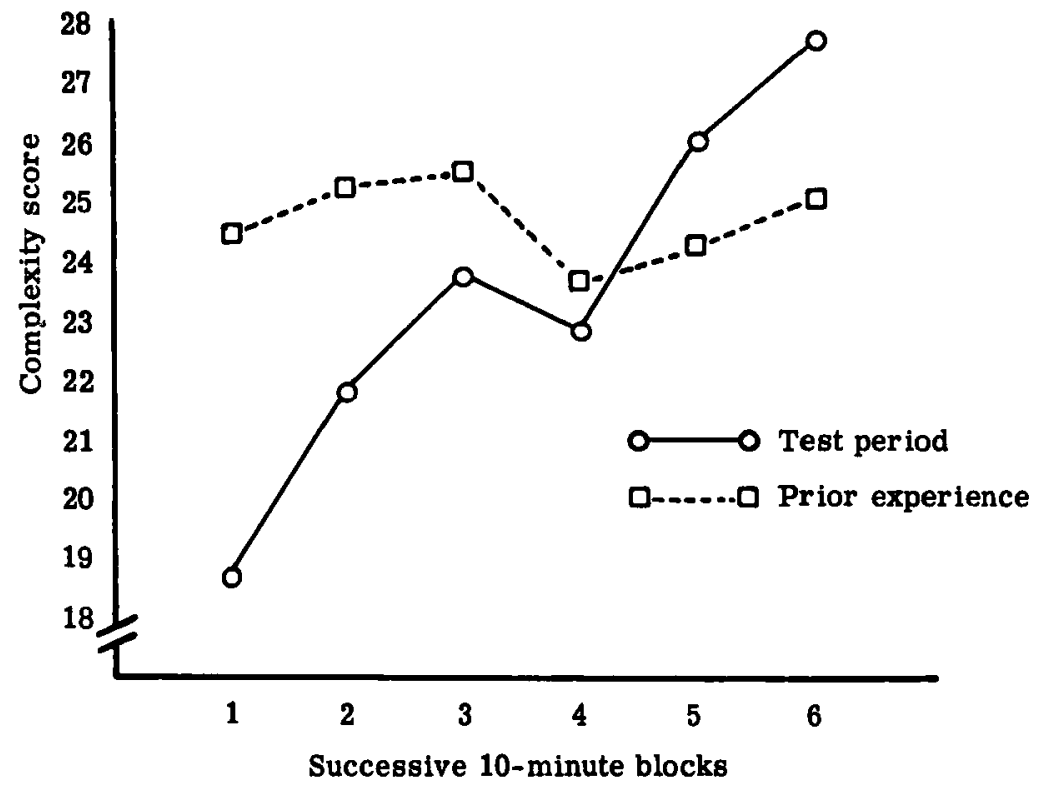

FIGURE 17. Changes in stimulus preference with time. 


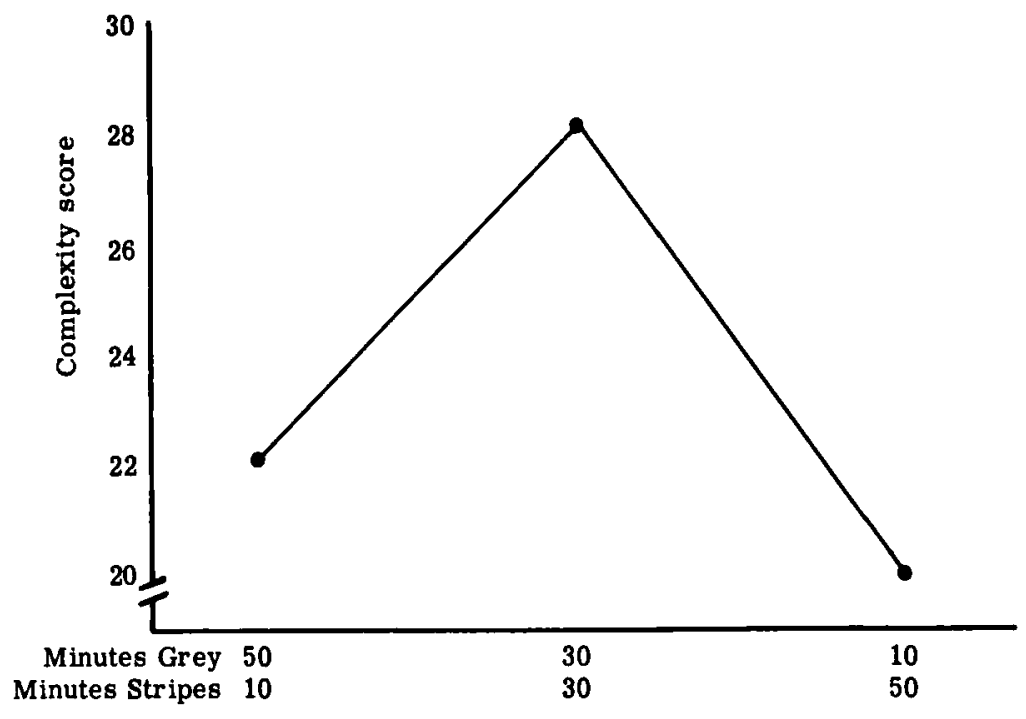

Character of prior experience

Figure 18. Generalized effects of stimulus exposure.

30 minutes and to the stripes for 30 minutes. The long exposure group was exposed to grey for ten minutes and to the stripes for 50 minutes.

At the end of the hour for each group, the 3/4-inch stripes were removed and the other four stripe patterns were introduced, one in each of the quadrants of the maze. The animals were then replaced, and their choices over a period of an hour were recorded. There were six animals in a group, a total of 18 in the experiment.

Complexity scores were again achieved by assigning arbitrary values to the stimuli ranging from a value of 1 to the widest stimulus pattern and a value of 4 to the narrowest. This value is then multiplied by the number of minutes in each tenminute block spent in each compartment. The scores could thus range from a minimum of 10 to a maximum of 40 .

FIGURE 17 shows the three groups combined to stress the progressive increase in preference for the more complex patterns over the hour of test compared to the exposure hour. FIGURE 18 replots the data to stress the differential effects of the exposure on the three groups.

The results are, in part, expected from complexity theory and, are in part, quite at variance with the expectation. During the one-hour test period, there is a progressive change in preference upward on the complexity dimension. The shift of nearly 10 points in FIGURE 17 indicates a shift of about one whole step across the "big" interval. In the first ten minutes the predominant preference was for the compartment with the $1 \& 1 / 2$-inch stripes, and in the last ten minutes, the predominant preference is for the compartment with the 3/8-inch stripes. This finding is in agreement with complexity theory.

The finding portrayed in FIGURE 18 is not easily explicable from the theory. The animals exposed to the 3/4-inch stripes for 30 minutes show a greater preference for more complex stimuli than does either the group exposed for ten minutes or the group exposed for $\mathbf{5 0}$ minutes. The locus of the problem may lie in small part in the wide gap between $3 / 8$ inches and $1 \& 1 / 2$-inches, created when 
TABLE 9

First-Order Transitional Proba bilities

\begin{tabular}{|c|c|c|c|c|c|}
\hline \multicolumn{6}{|c|}{ Short Exposure Group } \\
\hline FROM & $\begin{array}{l}3 \text { in. } \\
11 / 2 \text { in. } \\
3 / 8 \text { in. } \\
3 / 16 \text { in. }\end{array}$ & $\begin{array}{l}3 \text { in. } \\
.27 \\
.00 \\
.13 \\
.00\end{array}$ & $\begin{array}{c}11 / 2 \text { in. } \\
.03 \\
.03 \\
.03 \\
.00\end{array}$ & $\begin{array}{l}36 \text { in. } \\
.13 \\
.03 \\
.10 \\
.07\end{array}$ & $\begin{array}{c}\text { Y/6 in. } \\
.07 \\
.03 \\
.03 \\
.03\end{array}$ \\
\hline \multicolumn{6}{|c|}{ Middle Exposure Group } \\
\hline FROM & $\begin{array}{l}3 \mathrm{in} . \\
11 / 2 \mathrm{in} . \\
3 / 8 \mathrm{in.} \\
3 / 16 \mathrm{in} .\end{array}$ & $\begin{array}{l}.00 \\
.00 \\
.00 \\
.03\end{array}$ & $\begin{array}{l}.00 \\
.00 \\
.03 \\
.07\end{array}$ & $\begin{array}{l}.07 \\
.03 \\
.13 \\
.00\end{array}$ & $\begin{array}{l}.03 \\
.07 \\
.03 \\
.50\end{array}$ \\
\hline \multicolumn{6}{|c|}{ Long Exposure Group } \\
\hline FROM & $\begin{array}{l}3 \text { in. } \\
11 / 2 \text { in. } \\
3 / 8 \text { in. } \\
3 / 16 \text { in. }\end{array}$ & $\begin{array}{l}.27 \\
.10 \\
.07 \\
.00\end{array}$ & $\begin{array}{l}.03 \\
.10 \\
.07 \\
.00\end{array}$ & $\begin{array}{l}.17 \\
.03 \\
.00 \\
.03\end{array}$ & $\begin{array}{l}.07 \\
.03 \\
.00 \\
.03\end{array}$ \\
\hline
\end{tabular}

the middle stimulus was used for exposure, but why the three groups should react to that gap as they did is not clear.

The first-order transitional probabilities for the three groups are contained in TABLE 9. If one compares the probabilities in the diagonals immediately adjacent to the main diagonal with the three values in each case in the lower left and upper right corners, there is some support for the "gap" theory. The outer corners show higher probabilities than the adjacent diagonals. This is the reverse of the finding in the previous Tables. It indicates a tendency to go from one extreme to the other, rather than from one stimulus to one that is adjacent on the a priori scale.

One might conclude from this analysis that the animals were not reacting to the stimuli as representing four values on a single dimension. However, since the progression from simple to complex is quite clear, one would argue from that evidence that they were reacting to the stimuli as if on a complexity scale. The only post hoc explanation that has emerged is that the gap creates a situation in which the animals react as if there were only two points on the scale, narrow and wide. A convincing resolution of this finding lies in the future.

\section{Effect of Stimulus Range on Complexity and Preference I}

In the studies of judged complexity and preference in tartan patterns discussed earlier in this report, human subjects tended to respond to the range of stimuli presented to them. If they had a narrow range of stimuli on the less complex end of the scale, they tended to judge the upper end of their stimulus set as more complex than a group with the full range. If subjects judged only a set of more complex stimuli, they tended to judge the middle absolute range as less complex than subjects with the full range. From a variety of studies, it seemed possible that rats also responded to the range of stimuli presented to them rather than to responding on an absolute basis.

19 This study was carried out by Wade Boykin as a masters level research project, 19681969. It resulted in a paper entitled The Effects of Level and Range of Complexity Values on Visual Preferences in Rats. 
Boykin divided 18 (naive, male, Long Evans) rats at about 100-150 days of age into three groups of six animals each. One group (A) was to be exposed to a narrow range of relatively simple stimuli, a second group (B) was to be exposed to a narrow range of stimuli of high complexity, and a third group (C) was to be exposed to the full range. The stimuli selected on a priori grounds along with the items used with each of the three groups were the following:

Simple end

Complex end 2-inch horizontal stripes

2-inch vertical stripes

4 checkers per $4 \times 4$ square

9 checkers per $4 \times 4$ square

16 checkers per $4 \times 4$ square

25 checkers per $4 \times 4$ square

49 checkers per $4 \times 4$ square

81 checkers per $4 \times 4$ square

144 checkers per $4 \times 4$ square

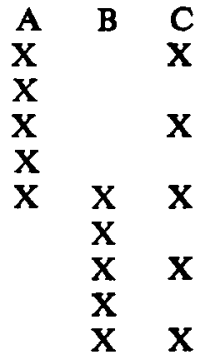

The experimental apparatus was a circular device with a center compartment from which radiated six doors permitting access, from the center, to six equally sized truncated pie-shaped compartments. These compartment walls were lined with the stimuli.

There were six compartments and five stimuli. One compartment was utilized as an entrance compartment. When the animal left the entrance compartment, it was closed behind him so that he had access during the trial to five stimulus compartments and the central compartment.

A trial consisted of ten minutes in the maze for 12 consecutive days for each animal.

The behavior of the animal was recorded on six of the channels of an EsterlineAngus recorder by marking where the animal was at all times during the trial as it activated the appropriate event marker.

FIGURE 19 shows the total amount of time spent by each group in each of

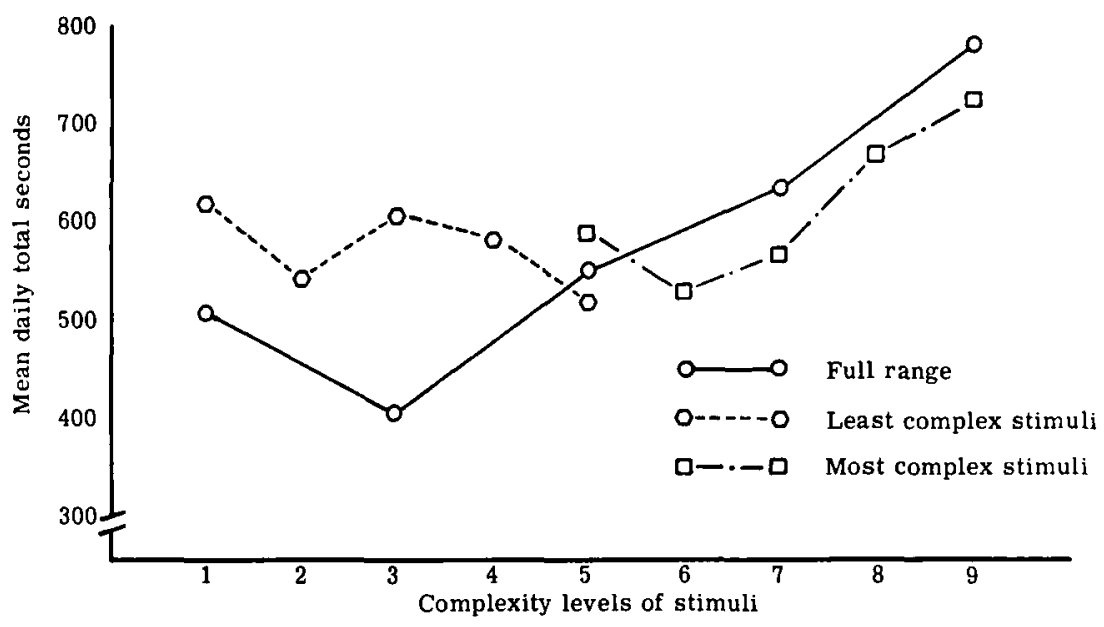

FIGURE 19-The effect of stimulus range on preference. 
the available stimulus compartments. Those in the low range of complexities do not show a preference. Those in the higher range show some preference for the more complex stimuli. The group exposed to the full range show a definite preference for the more complex stimuli. Thus the range of stimuli available did have an effect. Preference was more clearly expressed with the wider range of stimuli.

\section{Frequency versus Duration of Response as a Function of Stimulus}

When a set of stimuli is available for choice in the sense that the organism can see all the stimuli and choose among them, it is difficult to separate the effects of complexity on the frequency of choice from the effects on duration of choice. The necessity of distinguishing the factors determining the termination of a response from those that govern the next choice has been pointed out elsewhere. ${ }^{7}$ The concept of action decrement ${ }^{6}$ can be used to account for the termination of a response. Stimulus complexity may be a factor in delaying termination and, thus, in controlling the duration. Optimal stimulus levels should produce the maximum duration, and stimuli either more or less than optimum should produce shorter durations.

A test of this possibility has been devised by Stephen Sales. ${ }^{5}$ He constructed a box that consisted of a compartment that was about a foot in each dimension and that was totally dark when the lid was in place. On one side of the box there was a small hole through which a rat was able to stick his head far enough to break a photoelectric cell beam, thus allowing itself to see what was in the adjacent compartment. The adjacent compartment was arranged with a translucent panel positioned about 5 inches from the hole at floor level and slanted slightly toward the animal at the top. This panel was backlighted. The photoelectric cell was used to record the behavior of the rat.

The panel was replaceable. Stimulus complexity was varied by constructing nine such panels. Each consisted of a checkered pattern and they differed in the number of elements $2,4,9,16,25,64,100,196$, and 256.

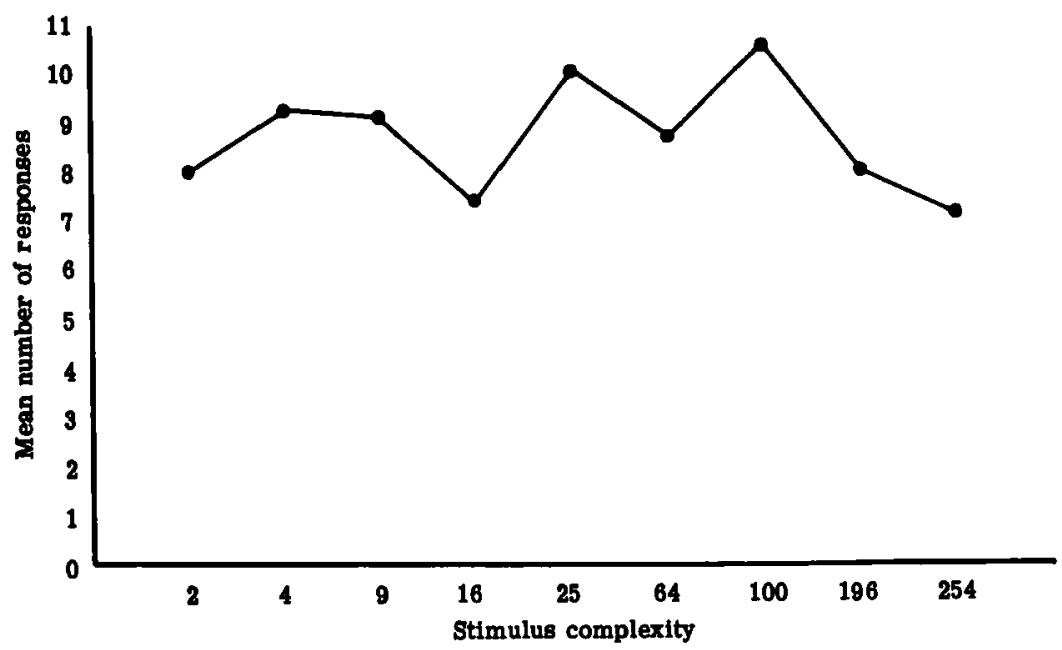

Figure 20. Frequency of response as a function of stimulus complexity. 


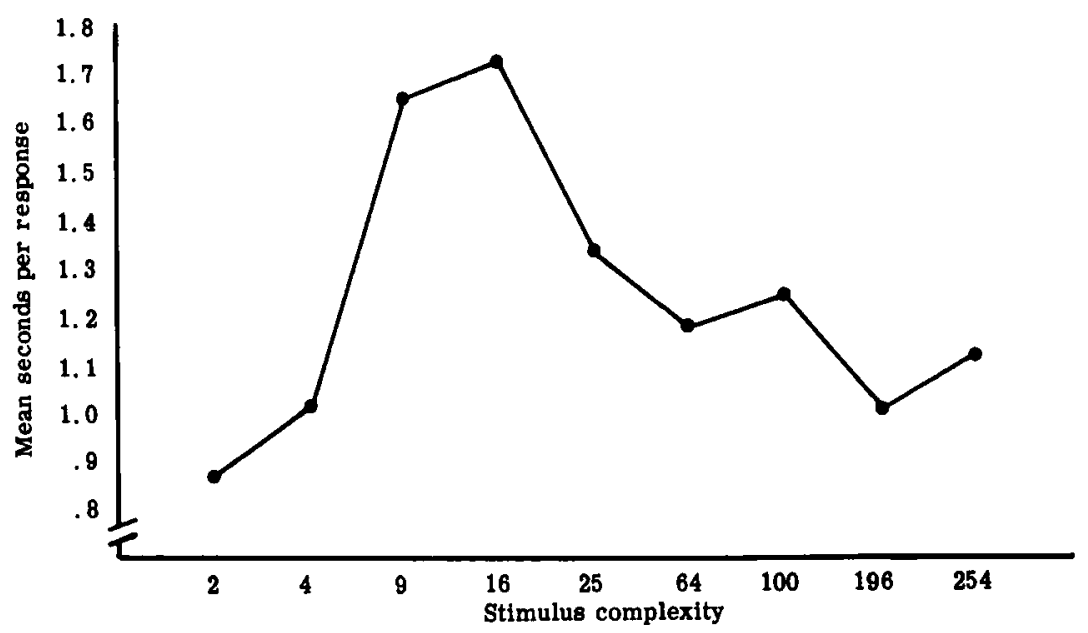

Figure 21. Duration of response as a function of stimulus complexity.

Nine groups of 15 rats each (total 135,108 male and 27 female) were each handled extensively and then introduced individually into the box. Ten seconds after introduction, the light was turned on in the adjacent compartment. Each animal was left in the box for a total of eight minutes. Each animal was tested only once and with a single stimulus.

The photocell recorded the frequency of response through a counter, and the total duration of responding through a clock that accumulated the total amount of time the animal's head was in a position to break the photobeam.

FIGURE 20 shows the frequency of responding in the nine groups thus selfexposed to the nine levels of stimulus complexity. There appears to be no orderly relationship between complexity and frequency. This result might be expected, since the animal could not see the stimulus until he responded.

FIGURE 21 shows the average duration of the responses, calculated by dividing the total response time by the frequency. Here, there is a clear optimum with stimuli around 9 and 16 elements. Thus, the complexity of the stimulus affected the duration of the response, with optimum complexity producing the longer responses, and either simpler or more complex stimuli producing responses of lesser durations.

\section{Summary of Animal Studies of Complexity and Preference}

The major finding of the animal studies of complexity and preference is that animals do tend to show preferences for stimuli of progressively increasing complexity with increased exposure in the choice situation. A correlated finding is that when the animal could not see the stimulus until he made a response, stimulus complexity increased the duration of the response. This finding is interesting in a context in which it is important to distinguish between factors that affect the termination of a psychological event and those that affect the choice of the next event.

Analysis of sequences of responses permitted the scaling of disparate stimuli and confirmed the fact that the animals were indeed responding to the order of the stimuli along a complexity scale. Animals were found to respond to the range of 
stimuli available, as had been found in the human studies. An attempt to demonstrate the predicted effect of prior exposure to a stimulus on a complexity dimension on subsequent choices yielded clear, but inexplicable, results.

\section{REFERENCES}

1. Berlyne, D. E. 1960. Conflict, Arousal and Curiosity. McGraw-Hill. New York.

2. Dember, W. N. 1960. The Psychology of Perception. Henry Holt and Co. New York.

3. Heвв, D. O. 1949. The Organization of Behavior. John Wiley and Sons, Inc. New York.

4. INNES, T. 1938. The Tartan of the Clans and Families of Scotland. Johnston. Edinburg and London.

5. Sales, S. M. 1968. Stimulus complexity as a determinant of approach behavior and inspection time in the hooded rat. Canad. J. Psychol./Rev. Canad. Psychol. 22: 11-17.

6. Walker, E. L. 1958. Action decrement and its relation to learning. Psychol. Rev. 65: 129-142.

7. Walker, E. L. 1964. Psychological complexity as a basis of a theory of motivation and choice. In Nebraska Symposium on Motivation. Levine, D., Ed. University of Nebraska Press. Lincoln, Neb.

8. WALkER, E. L. \& B. E. Walker. 1964. Response to stimulus complexity in the rat. Psychol. Rec. 14: 489-497.

\section{Discussion}

SYDNEY Vernon (Willimantic, Conn.): Dr. Walker indicated that art students accepted a greater degree of complexity than history and law students. I venture to submit that since these stimuli were visual, the art students had greater skills, and possibly, if the stimuli had been auditory, the history students might have accepted greater complexity, and if the stimuli had to do with social or interpersonal relationships, maybe your law students would have done better. Dr. Walker also indicated that certain sets of stimuli were satisfying. Now this implies a visceral reaction, and I submit that the whole perceptive mechanism doesn't involve merely the central nervous system, but comes from a combination of mind and body. To extend the concept that mind and body are part of the perceptive mechanism, I'd like to refer to the simple example of ulcers. Here, the flood of stimuli in the person who has ulcers gets to the brain, jumps over into his vegas, stimulates the stomach, pours out hydrochloric acid, and digests his mucosa.

The term "noise" was used, which always bothers me in scientific discussion. It seems to me that what is noise to one person may not be to another. The absence of noise means that the stimuli were meaningful, and if the stimuli were not meaningful, well then we use the term "noise." Of course I'm not speaking of the tremendous number of decibels that assail our accoustic organs, but, rather, scientifically. In his experiments with mice, and in dealing with the problem of assigning values to their peculiar behavior, Dr. Walker indicated that in those compartments that had colors, the grey, the stripes, and the checkered, the mice had a tendency to congregate in the grey area, and I submit that they did it because they felt secure there. Then later on the mice seemed to choose the battle area more and I submit that that was one time where they wanted privacy more than anything. Of course, all living creatures want to feel safe and want to feel important. Among other desires, they want variety, and that's why I think some of the mice stuck their heads through those holes.

And the degree of curiosity, or vitality, that Dr. Walker indicated the mice had possibly had something to do with the length of time they held their heads through those holes. 
DR. WALKER: Well, let me try to respond to your comments. Concerning the experiment having to do with experience, I agree with you. I apparently didn't make myself clear in trying to shorten this. The point $I$ was trying to make with respect to the experience of the art students was that it did have precisely the effect that you suggest. Because they had had more experience with visual stimuli, they were chosen. The law students were chosen because they had had less experience with visual stimuli. There were differences in the way in which they rated complexity, which was a result of their experience. While the data did not statistically demonstrate the optimal complexity shift upward for those subjects, it did demonstrate that they did react differently to the stimuli along the dimension that we were working with.

Now I should not have used the word noise. I was using it in the sense of "variance or error," a term that is referred to as "noise." This is not in my paper. In addition, I don't think I'll quibble with you over the language you choose to use with respect to the subject seeking that stimulus which represents his optimal complexity value. When you retranslate it, you use the word "security." This is acceptable. I used the word "preference."

DR. Moore (Brookdale Hospital): There's been a great deal of work on complexity and creativity complexity, and intelligence complexity, and neuroticism. The material you presented seems to undercut the idea of individual differences in complexity and preference. Could you comment on this?

DR. WALKER: My experiments are not intended to undercut that idea. As a matter of fact, I think there are enormous individual differences. What we're trying to do is find the major dimension of individual differences. And this is the reason for the distinction between stimulus complexity and/or psychological complexity, where the individual's reaction is expected to be different. That is why, in all these studies, even with the rats, we try to carry out the study in such a manner that you can analyze the data of an individual subject independently of the others. And had we had time, I could have shown you that there were indeed individual difference in rats, one of which Rat $Y$, scaled those four stimuli differently from the other two that did. I feel that this is the major focus of the theory. Trying to find a way to dimensionalize, get your hands on a major dimension which does describe individual differences. 